Supporting Information for:

\title{
Deoxygenative Hydrogenation of Amides Catalyzed by a Well-Defined Iridium Pincer Complex
}

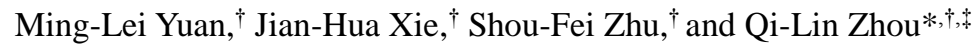 \\ $†$ State Key Laboratory and Institute of Elemento-organic Chemistry, Nankai University, Tianjin 300071, China \\ $\$$ Collaborative Innovation Center of Chemical Science and Engineering (Tianjin), Tianjin 300071, China
}

Corresponding Author:

*E-mail: qlzhou@ nankai.edu.cn

\section{CONTENTS:}

1. A Typical Procedure for Catalytic Hydrogenation of Amides

2. Complementary Reaction Optimization Data..............................................S3

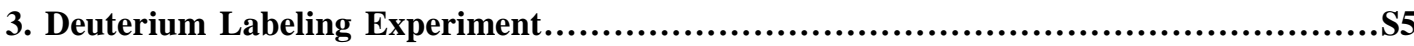

4. Hydrogenation of Amides Using Substoichiometric Amount of $B\left(\mathrm{C}_{6} \mathrm{~F}_{5}\right)_{3} \ldots \ldots \ldots \ldots \ldots \ldots . . . . . . . . . . .55$

5. Analytical Data of the Hydrogenation Products.........................................6

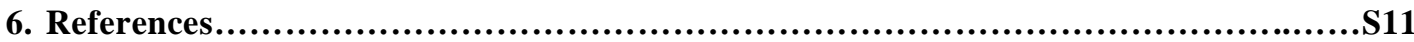

7. GC and NMR Spectra of the Hydrogenation Products......................................S13

General: Unless otherwise mentioned, all manipulations were performed in an argon-filled glove box (MBRAUN labstar) or using standard Schlenk techniques. NMR spectra were recorded on a Bruker AV 400 spectrometer at $400 \mathrm{MHz}\left({ }^{1} \mathrm{H} \mathrm{NMR}\right), 101 \mathrm{MHz}\left({ }^{13} \mathrm{C} \mathrm{NMR}\right)$ and $162 \mathrm{MHz}\left({ }^{31} \mathrm{P} \mathrm{NMR}\right)$. Chemical shifts were reported in ppm down field from internal $\left(\mathrm{CH}_{3}\right)_{4} \mathrm{Si}$ and external $85 \% \mathrm{H}_{3} \mathrm{PO}_{4}$, respectively. Data are presented in the following space: chemical shift, multiplicity, coupling constant in hertz $(\mathrm{Hz})$, and signal area integration in natural numbers. GC analyses were performed using a Hewlett Packard Model HP 7890 Series instruments. GCMS analysis was conducted on a GC System 7820A (G4320) connected to a MSD block 5977E (G7036A) using Agilent High Resolution Gas Chromatography Column.

Hydrogen gas (99.999\%) was purchased from Boc Gas Inc., Tianjin. All the solvents used for reactions were distilled under argon after drying over an appropriate drying agent. Iridium complexes $\mathbf{4} \mathbf{a}^{1}, \mathbf{4} \mathbf{b}^{2}, \mathbf{4} \mathbf{c}^{3}$, $\mathbf{5} \mathbf{a}^{4}, \mathbf{5} \mathbf{b}^{5}, \mathbf{6 a}^{6}, \mathbf{6 b}^{7}$ was prepared according to the literature procedure and kept in an argon-filled glove box. $\mathrm{B}\left(\mathrm{C}_{6} \mathrm{~F}_{5}\right)_{3}$ was purchased from TCI (Shanghai) Development Co,, Ltd. and sublimed at $80{ }^{\circ} \mathrm{C}$ under high vacuum before use. Other commercially available reagents were purchased from Acros, Strem Chemicals and Alfa Aesar Chemical Company. All of the amides for hydrogenation were recrystallized before use. 


\section{A Typical Procedure for Catalytic Hydrogenation of Amides}

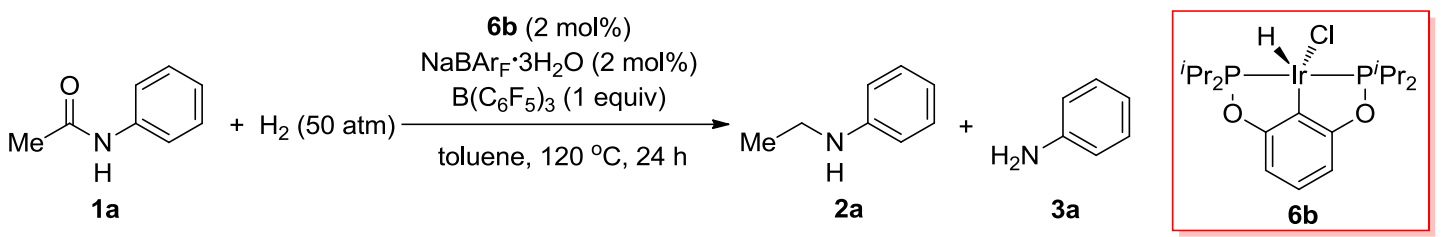

In a glove box, a glass vessel was charged with complex $\mathbf{6 b}(1.2 \mathrm{mg}, 2 \mu \mathrm{mol}), \mathrm{NaBAr}_{\mathrm{F}} 3 \mathrm{H}_{2} \mathrm{O}(2 \mathrm{mg}, 2$ $\mu \mathrm{mol})$, amide $(0.1 \mathrm{mmol})$ and $\mathrm{B}\left(\mathrm{C}_{6} \mathrm{~F}_{5}\right)_{3}(0.1 \mathrm{mmol})$. The reaction tube was placed into an autoclave and was added degassed toluene $(0.5 \mathrm{~mL})$ under argon atmosphere. The autoclave was purged three times with hydrogen and finally charged to $50 \mathrm{~atm}$. The reaction mixture was stirred at $120^{\circ} \mathrm{C}$ for $24 \mathrm{~h}$, then was cooled to room temperature. The pressure was released, and triphenylamine was added as an internal standard. The mixture was filtered through a short silica column and submitted to analysis of yield and selectivity of the reaction by GC. (Agilent DB-35, $30 \mathrm{~m} \times 0.32 \mathrm{~mm} \times 0.25 \mathrm{~mm}$ ) Using a flame ionization detector (FID) operating at $250{ }^{\circ} \mathrm{C}$. Injector temperature was set at $230{ }^{\circ} \mathrm{C}$. The carrier gas was nitrogen with a flow rate of $1.0 \mathrm{~mL} / \mathrm{min}$. The following temperature program was used in the analysis: $50{ }^{\circ} \mathrm{C}$ $10^{\circ} \mathrm{C} / \mathrm{min}-300{ }^{\circ} \mathrm{C}(10 \mathrm{~min})$. Using these conditions, the following retention times were observed: aniline (8.136 min), $N$-ethylaniline (10.005 $\mathrm{min}), \mathrm{N}$-phenylacetamide (14.316 min), triphenylamine (21.251 $\mathrm{min})$. The GC yield in this case is $89 \%$ and selectivity is $32: 1$. 


\section{Complementary Reaction Optimization Data}

Table S1. Investigation of Different Lewis Acids for Hydrogenation of Acetanil

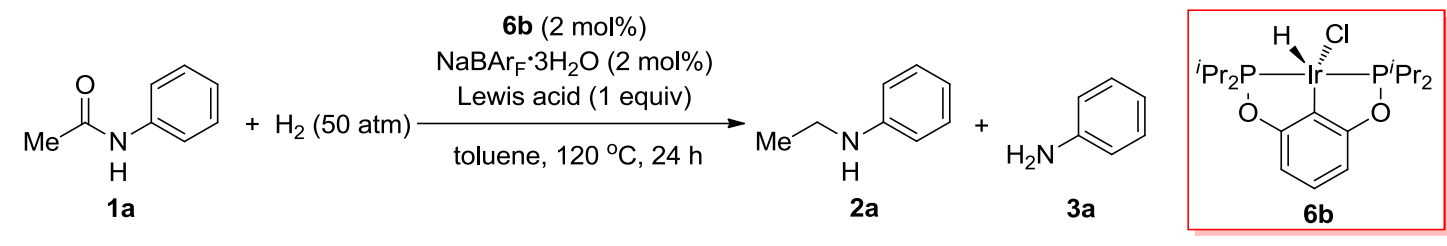

\begin{tabular}{|c|c|c|c|c|}
\hline Entry $^{a}$ & Lewis acid & Conv. $(\%)^{b}$ & Yield $(\%)^{b}$ & $\begin{array}{l}\text { Selectivity } \\
(\mathbf{2 a} / \mathbf{3 a})^{b}\end{array}$ \\
\hline 1 & $\mathrm{BEt}_{3}{ }^{c}$ & 70 & 62 & $19: 1$ \\
\hline 2 & $\mathrm{~B}\left(\mathrm{C}_{6} \mathrm{~F}_{5}\right)_{3}$ & 92 & 89 & $32: 1$ \\
\hline 3 & $\mathrm{BPh}_{3}$ & 2 & 2 & $100: 0$ \\
\hline 4 & $\mathrm{BF}_{3} \cdot \mathrm{Et}_{2} \mathrm{O}$ & 0 & NR & - \\
\hline 5 & $\mathrm{BCl}_{3}^{d}$ & 63 & 63 & $100: 0$ \\
\hline 6 & $\mathrm{BBr}_{3}^{e}$ & 18 & 16 & $8: 1$ \\
\hline 7 & $\mathrm{~B}\left(\mathrm{OSiMe}_{3}\right)_{3}$ & 10 & 9 & $10: 1$ \\
\hline 8 & $\mathrm{~B}(\mathrm{OMe})_{3}$ & 0 & NR & - \\
\hline 9 & $\mathrm{AlCl}_{3}$ & 0 & NR & - \\
\hline 10 & $\mathrm{AlMe}_{3}$ & 2.5 & 2.5 & $100: 0$ \\
\hline 11 & $\mathrm{AlEt}_{3}$ & 5 & 5 & $100: 0$ \\
\hline 12 & $\mathrm{EtAlCl}_{2}$ & 11 & 10 & $7.5: 1$ \\
\hline 13 & $\mathrm{TiCl}_{4}$ & 2 & 2 & $100: 0$ \\
\hline 14 & $\operatorname{Ti}\left(\mathrm{O}^{i} \mathrm{Pr}\right)_{4}$ & 14 & 10 & $2.8: 1$ \\
\hline 15 & $\operatorname{In}(\mathrm{OTf})_{3}$ & 35 & 0 & $0: 100$ \\
\hline 16 & $\mathrm{Sc}(\mathrm{OTf})_{3}$ & 0 & NR & - \\
\hline 17 & $\mathrm{Yb}(\mathrm{OTf})_{3}$ & 2.7 & 2.7 & $0: 100$ \\
\hline $18^{f}$ & $\mathrm{~B}\left(\mathrm{C}_{6} \mathrm{~F}_{5}\right)_{3}$ & 0 & NR & - \\
\hline $19^{f}$ & $\mathrm{~B}\left(\mathrm{C}_{6} \mathrm{~F}_{5}\right)_{3}+0.1$ eq. $\mathrm{PhNHEt}$ & 0 & NR & - \\
\hline $20^{f}$ & $\mathrm{~B}\left(\mathrm{C}_{6} \mathrm{~F}_{5}\right)_{3}+0.1$ eq. POCOP Ligand & 0 & NR & - \\
\hline
\end{tabular}

${ }^{a}$ Reaction conditions: $0.1 \mathrm{mmol}$ acetanil (1a), $0.2 \mathrm{~mol} \%$ 6b, $0.2 \mathrm{mmol} \% \mathrm{NaBAr}_{\mathrm{F}} \cdot 3 \mathrm{H}_{2} \mathrm{O}, 0.1 \mathrm{mmol}$ Lewis acid, $0.5 \mathrm{~mL}$ toluene, $50 \mathrm{~atm} \mathrm{H}_{2}, 120{ }^{\circ} \mathrm{C}, 24 \mathrm{~h} .{ }^{b}$ Conversions, yields and selectivities were determined by $\mathrm{GC}$ analysis using triphenylamine as an internal standard. ${ }^{c} \mathrm{BEt}_{3}$ was used as a $1 \mathrm{M}$ solution in $n$ hexane. ${ }^{d} \mathrm{BCl}_{3}$ was used as a $1 \mathrm{M}$ solution in $n$-heptane. ${ }^{e} \mathrm{BBr}_{3}$ was used as a $1 \mathrm{M}$ solution in $\mathrm{CH}_{2} \mathrm{Cl}_{2} .{ }^{f}$ In the absence of $\mathbf{6} \mathbf{b}$ and $\mathrm{NaBAr}_{\mathrm{F}} 3 \mathrm{H}_{2} \mathrm{O}$. 
Table S2. Effects of temperature, hydrogen pressure and solvent

\begin{tabular}{|c|c|c|c|c|c|c|}
\hline $\mathrm{e}^{\stackrel{\mathrm{O}}{\mathrm{N}}} \begin{array}{c}\mathrm{N} \\
\mathrm{H} \\
1 \mathrm{a}\end{array}$ & $+\mathrm{H}_{2}(\mathrm{x}$ & $\begin{array}{r}\mathrm{NaB} / \\
\mathrm{B}( \\
\text { sol }\end{array}$ & $\begin{array}{l}(2 \mathrm{~mol} \%) \\
3 \mathrm{H}_{2} \mathrm{O}(2 \mathrm{~mol} \%) \\
\left.5_{3}\right)_{3}(1 \text { equiv) } \\
\mathrm{tt}, \mathrm{T}^{\circ} \mathrm{C}, 24 \mathrm{~h}\end{array}$ & $\begin{array}{c}\mathrm{N} \\
\mathrm{H} \\
2 \mathrm{a}\end{array}$ & $\mathrm{H}_{2} \mathrm{Na}$ & (lb \\
\hline Entry $^{a}$ & $p\left(\mathrm{H}_{2}\right)(\mathrm{atm})$ & $\mathrm{T}\left({ }^{\circ} \mathrm{C}\right)$ & Solvent & Conv. $(\%)^{b}$ & Yield $(\%)^{b}$ & $\begin{array}{l}\text { Selectivity } \\
(\mathbf{2 a} / \mathbf{3 a})^{b}\end{array}$ \\
\hline 1 & 50 & 120 & toluene & 92 & 89 & $32: 1$ \\
\hline 2 & 50 & 120 & 1,4-dioxane & 2 & 2 & $100: 0$ \\
\hline 3 & 50 & 120 & $n$-heptane & 97 & 93 & $28: 1$ \\
\hline 4 & 50 & 120 & DCE & 8 & 8 & $100: 0$ \\
\hline 5 & 30 & 120 & toluene & 50 & 49 & $48: 1$ \\
\hline 6 & 70 & 120 & toluene & 95 & 92 & $30: 1$ \\
\hline 7 & 50 & 100 & toluene & 42 & 41 & $40: 1$ \\
\hline 8 & 50 & 140 & toluene & 96 & 92 & $24: 1$ \\
\hline
\end{tabular}

${ }^{a}$ Reaction conditions: $0.1 \mathrm{mmol}$ acetanil (1a), $0.2 \mathrm{~mol} \%$ 6b, $0.2 \mathrm{mmol} \% \mathrm{NaBAr}_{\mathrm{F}} \cdot 3 \mathrm{H}_{2} \mathrm{O}, 0.1 \mathrm{mmol}$ $\mathrm{B}\left(\mathrm{C}_{6} \mathrm{~F}_{5}\right)_{3}, 0.5 \mathrm{~mL}$ toluene, 50 atm $\mathrm{H}_{2}, 120{ }^{\circ} \mathrm{C}, 24 \mathrm{~h} .{ }^{b}$ Conversions, yields and selectivities were determined by GC analysis using triphenylamine as an internal standard.

\section{Table S3. Effects of counteranions}
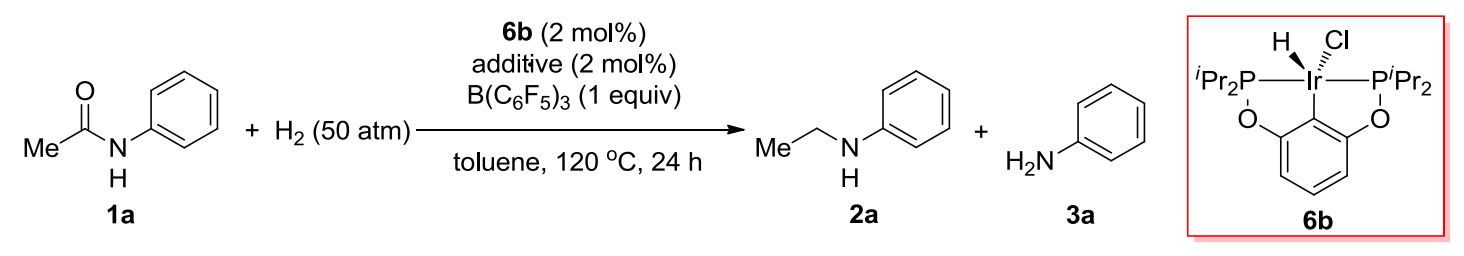

\begin{tabular}{lllll}
\hline Entry $^{a}$ & Additive & Conv. $(\%)^{b}$ & Yield $(\%)^{b}$ & $\begin{array}{l}\text { Selectivity } \\
(\mathbf{2 a} / \mathbf{3 a})^{b}\end{array}$ \\
\hline 1 & $\mathrm{NaBAr}_{\mathrm{F}}$ & 92 & 89 & $32: 1$ \\
2 & $\mathrm{NaBPh}_{4}$ & 76 & 70 & $13: 1$ \\
3 & $\mathrm{AgBF}_{4}$ & 49 & 47 & $26: 1$ \\
4 & $\mathrm{AgPF}_{6}$ & 45 & 34 & $8: 1^{[\mathrm{c}]}$ \\
5 & $\mathrm{AgSbF}_{6}$ & 42 & 41 & $53: 1$ \\
6 & AgOTf & 41 & 39 & $24: 1$ \\
\hline
\end{tabular}

${ }^{a}$ Reaction conditions: $0.1 \mathrm{mmol}$ acetanil (1a), $0.2 \mathrm{~mol} \% \mathbf{6 b}, 0.2 \mathrm{mmol} \%$ additive, $0.1 \mathrm{mmol} \mathrm{B}\left(\mathrm{C}_{6} \mathrm{~F}_{5}\right)_{3}$, $0.5 \mathrm{~mL}$ toluene, $50 \mathrm{~atm} \mathrm{H}_{2}, 120{ }^{\circ} \mathrm{C}, 24 \mathrm{~h} .{ }^{b}$ Conversions, yields and selectivities were determined by 
GC analysis using triphenylamine as an internal standard. ${ }^{c} 14 \%$ imine intermediate remained.

\section{Deuterium Labeling Experiment}

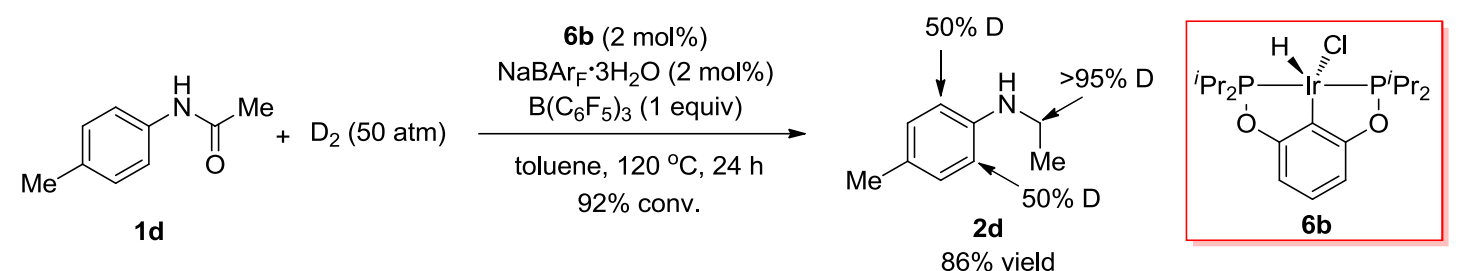

In a glove box, a glass vessel was charged with complex $6 \mathbf{b}(1.2 \mathrm{mg}, 2 \mu \mathrm{mol}), \mathrm{NaBAr}_{\mathrm{F}} 3 \mathrm{H}_{2} \mathrm{O}(2 \mathrm{mg}, 2$ $\mu \mathrm{mol})$, amide $(0.1 \mathrm{mmol})$ and $\mathrm{B}\left(\mathrm{C}_{6} \mathrm{~F}_{5}\right)_{3}(0.1 \mathrm{mmol})$. The reaction tube was placed into an autoclave and was added degassed toluene $(0.5 \mathrm{~mL})$ under argon atmosphere. The autoclave was purged three times with $\mathrm{D}_{2}$ and finally charged to $50 \mathrm{~atm}$. The reaction mixture was stirred at $120{ }^{\circ} \mathrm{C}$ for $24 \mathrm{~h}$, then was cooled to room temperature. Then, the pressure was released. The pure product was obtained by column chromatography on silica gel $(\mathrm{PE} / \mathrm{EA}=30: 1, \mathrm{v} / \mathrm{v})$ in $86 \%$ yield as a colorless oil.
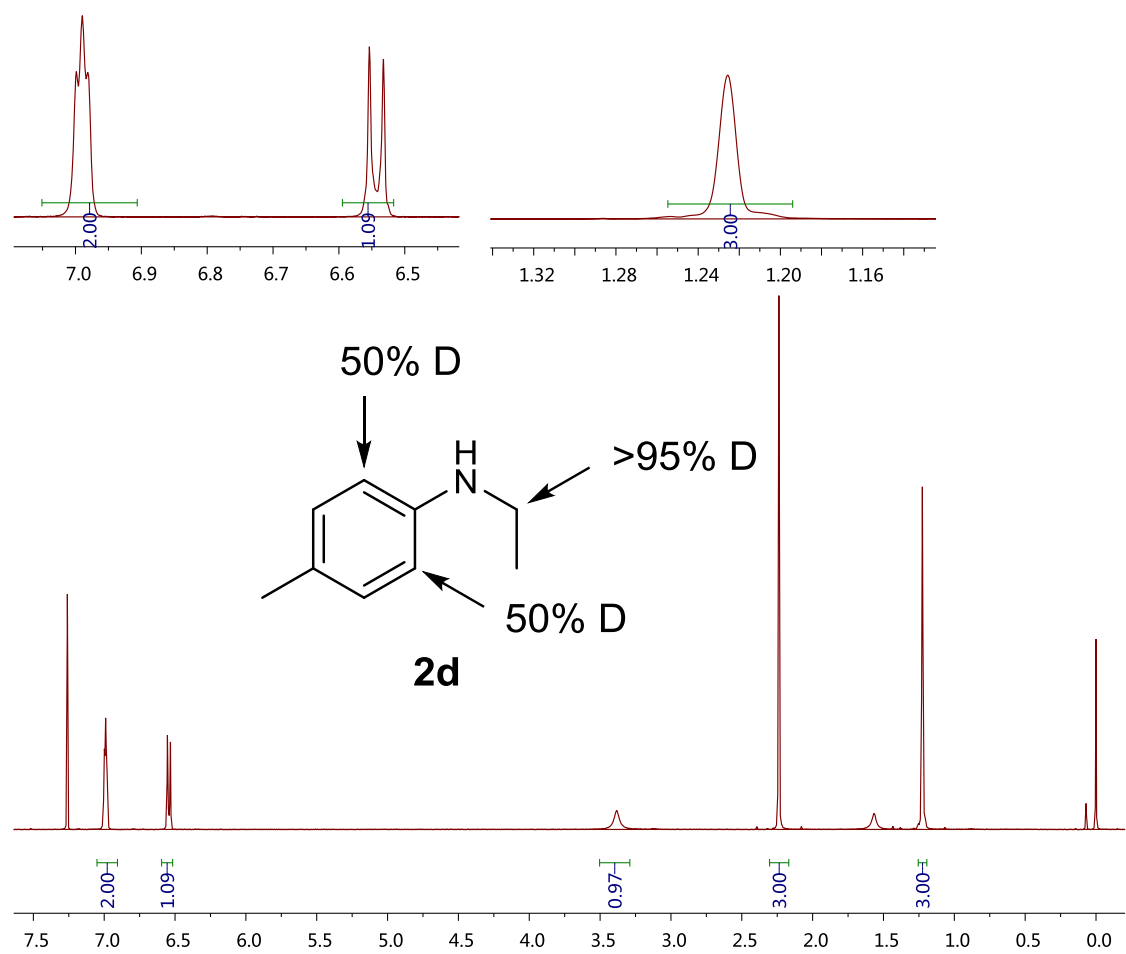

\section{Hydrogenation of Amides Using Substoichiometric Amount of $B\left(C_{6} F_{5}\right) 3^{8}$}

6b $(2 \mathrm{~mol} \%)$

$\mathrm{NaBAr}_{\mathrm{F}} 3 \mathrm{H}_{2} \mathrm{O}(2 \mathrm{~mol} \%)$

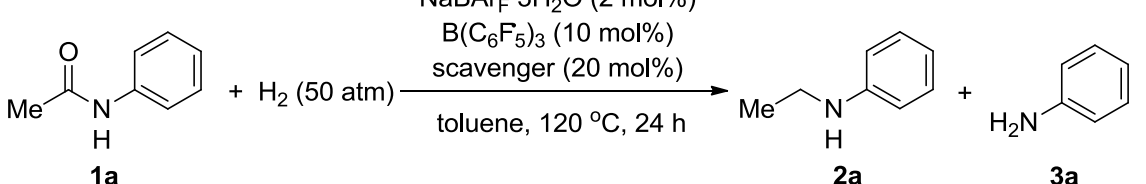

$3 a$

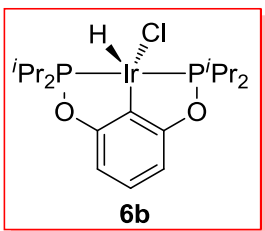

In a glove box, a glass vessel was charged with complex $6 \mathbf{b}(1.2 \mathrm{mg}, 2 \mu \mathrm{mol}), \mathrm{NaBAr}_{\mathrm{F}} 3 \mathrm{H}_{2} \mathrm{O}(2 \mathrm{mg}, 2$ $\mu \mathrm{mol})$, amide $(0.1 \mathrm{mmol})$ and $\mathrm{B}\left(\mathrm{C}_{6} \mathrm{~F}_{5}\right)_{3}(0.1 \mathrm{mmol})$. The reaction tube was placed into an autoclave and was added degassed toluene $(0.5 \mathrm{~mL})$ under argon atmosphere followed by adding the corresponding 
scavenger solution. The autoclave was purged three times with hydrogen and finally charged to $50 \mathrm{~atm}$. The reaction mixture was stirred at $120{ }^{\circ} \mathrm{C}$ for $24 \mathrm{~h}$, then was cooled to room temperature. The pressure was released, and triphenylamine was added as an internal standard. The mixture was filtered through a short silica column and submitted to analysis of yield and selectivity of the reaction by GC (Agilent DB$35,30 \mathrm{~m} \times 0.32 \mathrm{~mm} \times 0.25 \mathrm{~mm}$ ) using a flame ionization detector (FID) operating at $250{ }^{\circ} \mathrm{C}$. Injector temperature was set at $230^{\circ} \mathrm{C}$. The carrier gas was nitrogen with a flow rate of $1.0 \mathrm{~mL} / \mathrm{min}$. The following temperature program was used in the analysis: $50{ }^{\circ} \mathrm{C}-10^{\circ} \mathrm{C} / \mathrm{min}-300{ }^{\circ} \mathrm{C}(10 \mathrm{~min})$.

Table S4. Hydrogenation of Amides Using Substoichiometric Amount of $B\left(C_{6} F_{5}\right)_{3}$ and Scavengers. ${ }^{a}$

\begin{tabular}{lllll}
\hline Entry & Scavenger & Conv. $(\%)^{b}$ & ${\text { Yield }(\%)^{b}}^{b}$ & $\begin{array}{l}\text { Selectivity } \\
(\mathbf{2 a}: \mathbf{3 a})^{b}\end{array}$ \\
\hline 1 & - & 9 & 8.5 & $32: 1$ \\
2 & $\left({ }^{i} \mathrm{Bu}_{2} \mathrm{AlH}^{c}\right.$ & 49 & 39 & $4: 1$ \\
3 & $\mathrm{AlMe}^{d}$ & 55 & 46 & $5: 1$ \\
4 & $\mathrm{AlEt}_{3}{ }^{e}$ & 61 & 51 & $5: 1$ \\
5 & $\mathrm{Al}^{i} \mathrm{Bu}^{f}$ & 43 & $4: 1$ \\
6 & $\mathrm{ZnMe}_{2}{ }^{g}$ & 18 & 16 & $8: 1$ \\
7 & $\mathrm{ZnEt}_{2}{ }^{h}$ & 10 & 9 & $10: 1$ \\
8 & $4 \AA \mathrm{MS}^{i}$ & 34 & 32 & $29: 1$ \\
9 & $3 \AA \mathrm{MS}^{i}$ & 32 & 31 & $30: 1$ \\
10 & $4 \AA \mathrm{MS}^{i}{ }^{i}$ & 66 & 64 & $30: 1$ \\
\hline
\end{tabular}

${ }^{a}$ Reaction conditions: $0.5 \mathrm{mmol}$ acetanil (1a), $1 \mathrm{~mol} \%$ 6b, $1 \mathrm{mmol} \% \mathrm{NaBAr}_{\mathrm{F}} \cdot 3 \mathrm{H}_{2} \mathrm{O}, 0.05 \mathrm{mmol} \mathrm{B}\left(\mathrm{C}_{6} \mathrm{~F}_{5}\right)_{3}$, $2.5 \mathrm{~mL}$ toluene, $50 \mathrm{~atm} \mathrm{H}_{2}, 120{ }^{\circ} \mathrm{C}, 24 \mathrm{~h} .{ }^{b}$ Conversions, yields and selectivities were determined by $\mathrm{GC}$ analysis using triphenylamine as an internal standard. ${ }^{c}\left({ }^{i} \mathrm{Bu}\right)_{2} \mathrm{AlH}$ was used as a $1.0 \mathrm{M}$ solution in hexane. ${ }^{d} \mathrm{AlMe}_{3}$ was used as a $1.0 \mathrm{M}$ solution in heptane. ${ }^{e} \mathrm{AlEt}_{3}$ was used as a $0.6 \mathrm{M}$ solution in heptane. ${ }^{f} \mathrm{Al}\left({ }^{i} \mathrm{Bu}\right)_{3}$ was used as a $1.0 \mathrm{M}$ solution in hexane. ${ }^{g} \mathrm{ZnMe}_{2}$ was used as a $1.0 \mathrm{M}$ solution in toluene. ${ }^{h}$ $\mathrm{ZnEt}_{2}$ was used as a $1.0 \mathrm{M}$ solution in hexane. ${ }^{i}$ Using $100 \mathrm{mg}$ molecular sieve, $48 \mathrm{~h} .{ }^{j}$ sing $50 \mathrm{~mol} \%$ $\mathrm{B}\left(\mathrm{C}_{6} \mathrm{~F}_{5}\right)_{3}$.

\section{Analytical Data of the Hydrogenation Products}

\section{$N$-ethylaniline $(2 \mathbf{a})^{9}$}<smiles>CCNc1ccccc1</smiles>

Colorless oil, $10.3 \mathrm{mg}(85 \%),{ }^{1} \mathrm{H}$ NMR (400 MHz, $\left.\mathrm{CDCl}_{3}\right) \delta^{1} \mathrm{H}$ NMR (400 MHz, $\left.\mathrm{CDCl}_{3}\right) \delta 7.23-7.16(\mathrm{~m}, 2 \mathrm{H}), 6.71(\mathrm{td}, J=7.3,0.6 \mathrm{~Hz}, 1 \mathrm{H}), 6.62(\mathrm{~d}, J=8.1 \mathrm{~Hz}, 2 \mathrm{H})$, 3.54 (br s, 1H), 3.17 (q, $J=7.2 \mathrm{~Hz}, 2 \mathrm{H}), 1.27$ (t, $J=7.2 \mathrm{~Hz}, 3 \mathrm{H}) .{ }^{13} \mathrm{C}$ NMR (100 MHz, $\left.\mathrm{CDCl}_{3}\right) \delta 148.5,129.2,117.2,112.8,38.5,14.9$. GC conditions [Agilent DB-35, $30 \mathrm{~m}$ $\times 0.32 \mathrm{~mm} \times 0.25 \mathrm{~mm}$, injector temp. $230{ }^{\circ} \mathrm{C}$, detector temp. $250{ }^{\circ} \mathrm{C}, 50{ }^{\circ} \mathrm{C} \operatorname{ramps} 10{ }^{\circ} \mathrm{C} / \mathrm{min}$ to $300{ }^{\circ} \mathrm{C}$, hold $10 \mathrm{~min}, \mathrm{~N}_{2}$ fluency $1.0 \mathrm{~mL} / \mathrm{min}$. $t=8.136 \mathrm{~min}$ for aniline, $t=10.005 \mathrm{~min}$ for $\mathrm{N}$ ethylaniline, $t=14.316 \mathrm{~min}$ for acetanil, $t=21.251 \mathrm{~min}$ for triphenylamine.] 


\section{$N$-ethyl-2-methylaniline (2b) ${ }^{10}$}

Colorless oil, $11.0 \mathrm{mg}(83 \%),{ }^{1} \mathrm{H}$ NMR $\left(400 \mathrm{MHz}, \mathrm{CDCl}_{3}\right) \delta 7.14(\mathrm{t}, J=7.7 \mathrm{~Hz}, 1 \mathrm{H})$,<smiles>CCNc1ccccc1C</smiles>

$7.06(\mathrm{~d}, J=7.3 \mathrm{~Hz}, 1 \mathrm{H}), 6.70-6.59(\mathrm{~m}, 2 \mathrm{H}), 3.38$ (br s, 1H), 3.21 (q, $J=7.1 \mathrm{~Hz}, 2 \mathrm{H})$,

$2.15(\mathrm{~s}, 3 \mathrm{H}), 1.32(\mathrm{td}, J=7.1,0.9 \mathrm{~Hz}, 3 \mathrm{H}) .{ }^{13} \mathrm{C} \mathrm{NMR}\left(100 \mathrm{MHz}, \mathrm{CDCl}_{3}\right) \delta 146.4$, 130.0, 127.2, 121.7, 116.8, 109.6, 38.4, 17.5, 15.0. GC conditions [Agilent DB-35, 30 $\mathrm{m} \times 0.32 \mathrm{~mm} \times 0.25 \mathrm{~mm}$, injector temp. $230{ }^{\circ} \mathrm{C}$, detector temp. $250{ }^{\circ} \mathrm{C}, 50{ }^{\circ} \mathrm{C}$ ramps $10{ }^{\circ} \mathrm{C} / \mathrm{min}$ to $300{ }^{\circ} \mathrm{C}$, hold $10 \mathrm{~min}, \mathrm{~N}_{2}$ fluency $1.0 \mathrm{~mL} / \mathrm{min} . t=9.490 \mathrm{~min}$ for $o$-toluidine, $t=11.032 \mathrm{~min}$ for $N$ ethyl-2-methylaniline, $t=14.657 \mathrm{~min}$ for $N$-(o-tolyl $)$ acetamide, $t=21.294 \mathrm{~min}$ for triphenylamine.]

\section{$N$-ethyl-3-methylaniline (2c) $)^{11}$}

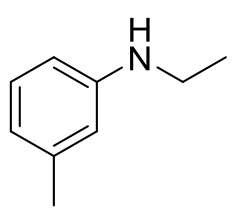

Colorless oil, $11.8 \mathrm{mg}(88 \%),{ }^{1} \mathrm{H}$ NMR $\left(400 \mathrm{MHz}, \mathrm{CDCl}_{3}\right) \delta 7.07$ (t, $\left.J=7.9 \mathrm{~Hz}, 1 \mathrm{H}\right)$, $6.53(\mathrm{~d}, J=7.4 \mathrm{~Hz}, 1 \mathrm{H}), 6.47-6.40(\mathrm{~m}, 2 \mathrm{H}), 3.49$ (br s, 1H), 3.16 (q, $J=7.1 \mathrm{~Hz}, 2 \mathrm{H})$, $2.31(\mathrm{~s}, 3 \mathrm{H}), 1.28(\mathrm{t}, J=7.1 \mathrm{~Hz}, 3 \mathrm{H}) .{ }^{13} \mathrm{C} \mathrm{NMR}\left(100 \mathrm{MHz}, \mathrm{CDCl}_{3}\right)$ 148.5, 139.0, 129.1, 118.2, 113.5, 109.9, 38.5, 21.6, 15.0. GC conditions [Agilent DB-35, $30 \mathrm{~m} \times$ $0.32 \mathrm{~mm} \times 0.25 \mathrm{~mm}$, injector temp. $230{ }^{\circ} \mathrm{C}$, detector temp. $250{ }^{\circ} \mathrm{C}, 50{ }^{\circ} \mathrm{C}$ ramps $10{ }^{\circ} \mathrm{C} / \mathrm{min}$ to $300{ }^{\circ} \mathrm{C}$, hold $10 \mathrm{~min}, \mathrm{~N}_{2}$ fluency $1.0 \mathrm{~mL} / \mathrm{min} . t=9.520 \mathrm{~min}$ for $m$-toluidine, $t=11.320$ min for $N$-ethyl-3-methylaniline, $t=14.657 \mathrm{~min}$ for $N$-( $m$-tolyl $)$ acetamide, $t=21.342 \mathrm{~min}$ for triphenylamine.]

\section{$N$-ethyl-4-methylaniline (2d) ${ }^{11}$}<smiles>CCNc1ccc(C)cc1</smiles>

Colorless oil, $12.2 \mathrm{mg}(91 \%),{ }^{1} \mathrm{H}$ NMR $\left(400 \mathrm{MHz}, \mathrm{CDCl}_{3}\right) 7.01(\mathrm{~d}, \mathrm{~J}=7.9 \mathrm{~Hz}, 2 \mathrm{H})$, $6.56(\mathrm{~d}, \mathrm{~J}=8.0 \mathrm{~Hz}, 2 \mathrm{H}), 3.41$ (br s, 1H), 3.15 (q, J = 7.1 Hz, 2H), 2.26 (s, 3H), 1.26 $(\mathrm{t}, \mathrm{J}=7.1 \mathrm{~Hz}, 3 \mathrm{H}) .{ }^{13} \mathrm{C} \mathrm{NMR}\left(100 \mathrm{MHz}, \mathrm{CDCl}_{3}\right) \delta 146.3,129.7,126.5,113.0,38.9$, 20.4, 15.0. GC conditions [Agilent DB-35, $30 \mathrm{~m} \times 0.32 \mathrm{~mm} \times 0.25 \mathrm{~mm}$, injector temp. $230{ }^{\circ} \mathrm{C}$, detector temp. $250{ }^{\circ} \mathrm{C}, 50{ }^{\circ} \mathrm{C}$ ramps $10{ }^{\circ} \mathrm{C} / \mathrm{min}$ to $300{ }^{\circ} \mathrm{C}$, hold $10 \mathrm{~min}, \mathrm{~N}_{2}$ fluency $1.0 \mathrm{~mL} / \mathrm{min}$. $t=9.444 \mathrm{~min}$ for $p$-toluidine, $t=11.211 \mathrm{~min}$ for $N$-ethyl-4-methylaniline, $t=15.456$ $\min$ for $N$-( $p$-tolyl)acetamide, $t=21.284$ min for triphenylamine.]

\section{$\mathrm{N}$-ethyl-2,4-dimethylaniline (2e) ${ }^{12}$}<smiles>CCNc1ccc(C)cc1C</smiles>

Colorless oil, $12.6 \mathrm{mg}(85 \%),{ }^{1} \mathrm{H}$ NMR $\left(400 \mathrm{MHz}, \mathrm{CDCl}_{3}\right) 6.99(\mathrm{~d}, J=8.1 \mathrm{~Hz}$, $1 \mathrm{H}), 6.89$ (s, 1H), 6.54 (d, $J=8.1 \mathrm{~Hz}, 1 \mathrm{H}), 3.28$ (br s, 1H), 3.22 (q, $J=7.1 \mathrm{~Hz}$, 2H), $2.29(\mathrm{~s}, 3 \mathrm{H}), 2.16(\mathrm{~s}, 3 \mathrm{H}), 1.34(\mathrm{t}, J=6.9 \mathrm{~Hz}, 3 \mathrm{H}) .{ }^{13} \mathrm{C}$ NMR $(100 \mathrm{MHz}$, $\left.\mathrm{CDCl}_{3}\right) \delta 144.1,130.9,127.3,125.9,121.9,109.9,38.7,20.4,17.4,15.1 . \mathrm{GC}$ conditions [Agilent DB-35, $30 \mathrm{~m} \times 0.32 \mathrm{~mm} \times 0.25 \mathrm{~mm}$, injector temp. $230{ }^{\circ} \mathrm{C}$, detector temp. $250{ }^{\circ} \mathrm{C}, 50{ }^{\circ} \mathrm{C}$ ramps $10{ }^{\circ} \mathrm{C} / \mathrm{min}$ to $300{ }^{\circ} \mathrm{C}$, hold $10 \mathrm{~min}, \mathrm{~N}_{2}$ fluency $1.0 \mathrm{~mL} / \mathrm{min} . t=10.732 \mathrm{~min}$ for 2,4-dimethylaniline, $t=12.160 \mathrm{~min}$ for $N$-ethyl-2,4-dimethylaniline, $t=15.960 \mathrm{~min}$ for $N$-(2,4dimethylphenyl)acetamide, $t=21.308 \mathrm{~min}$ for triphenylamine.]

\section{2-chloro- $N$-ethylaniline $(2 \mathrm{f})^{13}$}<smiles>CCNc1ccccc1Cl</smiles>

Colorless oil, $12.8 \mathrm{mg}(82 \%),{ }^{1} \mathrm{H}$ NMR $\left(400 \mathrm{MHz}, \mathrm{CDCl}_{3}\right) \delta 7.24(\mathrm{~d}, J=7.3 \mathrm{~Hz}, 1 \mathrm{H})$, $7.13(\mathrm{t}, J=7.7 \mathrm{~Hz}, 1 \mathrm{H}), 6.71-6.59(\mathrm{~m}, 2 \mathrm{H}), 4.18(\mathrm{br} \mathrm{s}, 1 \mathrm{H}), 3.32-3.11(\mathrm{~m}, 2 \mathrm{H}), 1.30$ $(\mathrm{t}, J=7.1 \mathrm{~Hz}, 3 \mathrm{H}) .{ }^{13} \mathrm{C} \mathrm{NMR}\left(100 \mathrm{MHz}, \mathrm{CDCl}_{3}\right) \delta 144.2,129.1,127.8,118.9,116.9$, 111.1, 38.2, 14.7. GC conditions [Agilent DB-35, $30 \mathrm{~m} \times 0.32 \mathrm{~mm} \times 0.25 \mathrm{~mm}$, injector 
temp. $230{ }^{\circ} \mathrm{C}$, detector temp. $250{ }^{\circ} \mathrm{C}, 50{ }^{\circ} \mathrm{C}$ ramps $10{ }^{\circ} \mathrm{C} / \mathrm{min}$ to $300{ }^{\circ} \mathrm{C}$, hold $10 \mathrm{~min}, \mathrm{~N}_{2}$ fluency $1.0 \mathrm{~mL} / \mathrm{min}$. $t=10.352 \mathrm{~min}$ for 2-chloroaniline, $t=11.654 \mathrm{~min}$ for 2-chloro- $N$-ethylaniline, $t=$ $14.318 \mathrm{~min}$ for $N$-(2-chlorophenyl)acetamide, $t=21.287 \mathrm{~min}$ for triphenylamine.]

\section{3-chloro- $N$-ethylaniline $(2 \mathrm{~g})^{14}$}<smiles>CCNc1cccc(Cl)c1</smiles>

Colorless oil, $13.1 \mathrm{mg}(84 \%),{ }^{1} \mathrm{H} \mathrm{NMR}\left(400 \mathrm{MHz}, \mathrm{CDCl}_{3}\right) \delta 7.06(\mathrm{t}, J=8.0 \mathrm{~Hz}, 1 \mathrm{H})$, $6.64(\mathrm{ddd}, J=7.9,1.9,0.9 \mathrm{~Hz}, 1 \mathrm{H}), 6.57(\mathrm{t}, J=2.1 \mathrm{~Hz}, 1 \mathrm{H}), 6.46(\mathrm{ddd}, J=8.2,2.2$, $0.7 \mathrm{~Hz}, 1 \mathrm{H}), 3.63$ (br s, $1 \mathrm{H}), 3.13(\mathrm{q}, J=7.1 \mathrm{~Hz}, 2 \mathrm{H}), 1.25$ (t, $J=7.2 \mathrm{~Hz}, 3 \mathrm{H}) .{ }^{13} \mathrm{C}$ NMR $\left(100 \mathrm{MHz}, \mathrm{CDCl}_{3}\right) \delta 149.5,135.0,130.1,117.0,112.2,111.1,38.3,14.7 . \mathrm{GC}$ conditions [Agilent DB-35, $30 \mathrm{~m} \times 0.32 \mathrm{~mm} \times 0.25 \mathrm{~mm}$, injector temp. $230{ }^{\circ} \mathrm{C}$, detector temp. $250{ }^{\circ} \mathrm{C}, 50{ }^{\circ} \mathrm{C}$ ramps $10{ }^{\circ} \mathrm{C} / \mathrm{min}$ to $300{ }^{\circ} \mathrm{C}$, hold $10 \mathrm{~min}, \mathrm{~N}_{2}$ fluency $1.0 \mathrm{~mL} / \mathrm{min} . t=$ $11.568 \mathrm{~min}$ for 3-chloroaniline, $t=13.113 \mathrm{~min}$ for 3-chloro- $N$-ethylaniline, $t=16.788 \mathrm{~min}$ for $\mathrm{N}$ (3-chlorophenyl)acetamide, $t=21.306 \mathrm{~min}$ for triphenylamine.]

\section{4-chloro- $N$-ethylaniline (2h) ${ }^{14}$}<smiles>CCNc1ccc(Cl)cc1</smiles>

Colorless oil, $13.7 \mathrm{mg}(88 \%),{ }^{1} \mathrm{H}$ NMR $\left(400 \mathrm{MHz}, \mathrm{CDCl}_{3}\right) \delta 7.11(\mathrm{~d}, J=8.6 \mathrm{~Hz}$, $2 \mathrm{H}), 6.52(\mathrm{~d}, J=8.6 \mathrm{~Hz}, 2 \mathrm{H}), 3.55(\mathrm{br} \mathrm{s}, 1 \mathrm{H}), 3.12(\mathrm{q}, J=7.1 \mathrm{~Hz}, 2 \mathrm{H}), 1.25(\mathrm{t}, J$ $=7.1 \mathrm{~Hz}, 3 \mathrm{H}) .{ }^{13} \mathrm{C}$ NMR $\left(100 \mathrm{MHz}, \mathrm{CDCl}_{3}\right) \delta 147.0,129.0,121.7,113.8,38.6$, 14.8. GC conditions [Agilent DB-35, $30 \mathrm{~m} \times 0.32 \mathrm{~mm} \times 0.25 \mathrm{~mm}$, injector temp. $230{ }^{\circ} \mathrm{C}$, detector temp. $250{ }^{\circ} \mathrm{C}, 50{ }^{\circ} \mathrm{C}$ ramps $10{ }^{\circ} \mathrm{C} / \mathrm{min}$ to $300{ }^{\circ} \mathrm{C}$, hold $10 \mathrm{~min}, \mathrm{~N}_{2}$ fluency 1.0 $\mathrm{mL} / \mathrm{min}$. $t=11.673 \mathrm{~min}$ for 2-chloroaniline, $t=13.233 \mathrm{~min}$ for 2 -chloro- $N$-ethylaniline, $t=16.782$ $\min$ for $N$-(2-chlorophenyl)acetamide, $t=21.299 \mathrm{~min}$ for triphenylamine.]

\section{4-bromo- $N$-ethylaniline $(2 \mathrm{i})^{15}$}<smiles>CCNc1ccc(Br)cc1</smiles>

Yellow oil, $14.6 \mathrm{mg}(73 \%),{ }^{1} \mathrm{H}$ NMR $\left(400 \mathrm{MHz}, \mathrm{CDCl}_{3}\right) \delta 7.24(\mathrm{~d}, J=8.6 \mathrm{~Hz}$, $2 \mathrm{H}), 6.47$ (d, $J=8.5 \mathrm{~Hz}, 2 \mathrm{H}), 3.57$ (br s, 1H), 3.11 (q, $J=7.1 \mathrm{~Hz}, 2 \mathrm{H}), 1.24$ (t, $J$ $=7.1 \mathrm{~Hz}, 3 \mathrm{H}) .{ }^{13} \mathrm{C} \mathrm{NMR}\left(100 \mathrm{MHz}, \mathrm{CDCl}_{3}\right) \delta 147.4,131.9,114.3,108.7,38.5$, 14.7. GC conditions [Agilent DB-35, $30 \mathrm{~m} \times 0.32 \mathrm{~mm} \times 0.25 \mathrm{~mm}$, injector temp. $230{ }^{\circ} \mathrm{C}$, detector temp. $250{ }^{\circ} \mathrm{C}, 50{ }^{\circ} \mathrm{C}$ ramps $10{ }^{\circ} \mathrm{C} / \mathrm{min}$ to $300{ }^{\circ} \mathrm{C}$, hold $10 \mathrm{~min}, \mathrm{~N}_{2}$ fluency 1.0 $\mathrm{mL} / \mathrm{min} . t=13.143 \mathrm{~min}$ for 4-bromoaniline, $t=14.557 \mathrm{~min}$ for 4 -bromo- $N$-ethylaniline, $t=18.165$ min for $N$-(4-bromophenyl)acetamide, $t=21.302 \mathrm{~min}$ for triphenylamine.]<smiles>CCNc1cccc(F)c1</smiles>

aniline $(2 \mathbf{j})^{16}$

Colorless oil,11.0 mg (78\%), ${ }^{1} \mathrm{H}$ NMR (400 MHz, $\left.\mathrm{CDCl}_{3}\right) \delta 7.14-7.03(\mathrm{~m}, 1 \mathrm{H}), 6.52$ - $6.10(\mathrm{~m}, 3 \mathrm{H}), 3.68(\mathrm{br} \mathrm{s}, 1 \mathrm{H}), 3.17-3.10(\mathrm{~m}, 2 \mathrm{H}), 1.26(\mathrm{t}, J=7.2 \mathrm{~Hz}, 3 \mathrm{H}) .{ }^{13} \mathrm{C} \mathrm{NMR}$ $\left(100 \mathrm{MHz}, \mathrm{CDCl}_{3}\right) \delta 164.2\left(\mathrm{~d},{ }^{1} J_{C-F}=242.4 \mathrm{~Hz}\right), 150.2\left(\mathrm{~d},{ }^{2} J_{C-F}=10.1 \mathrm{~Hz}\right), 130.2(\mathrm{~d}$, $\left.{ }^{3} J_{C-F}=10.1 \mathrm{~Hz}\right), 108.6,103.5\left(\mathrm{~d},{ }^{4} J_{C-F}=30.3 \mathrm{~Hz}\right), 99.2\left(\mathrm{~d},{ }^{5} J_{C-F}=30.3 \mathrm{~Hz}\right), 38.4,14.7$. GC conditions [Agilent DB-35, $30 \mathrm{~m} \times 0.32 \mathrm{~mm} \times 0.25 \mathrm{~mm}$, injector temp. $230^{\circ} \mathrm{C}$, detector temp. $250{ }^{\circ} \mathrm{C}, 50{ }^{\circ} \mathrm{C}$ ramps $10{ }^{\circ} \mathrm{C} / \mathrm{min}$ to $300{ }^{\circ} \mathrm{C}$, hold $10 \mathrm{~min}, \mathrm{~N}_{2}$ fluency $1.0 \mathrm{~mL} / \mathrm{min} . t=$ $8.668 \mathrm{~min}$ for 3-fluoroaniline, $t=10.381 \mathrm{~min}$ for 3-fluoro- $N$-ethylaniline, $t=14.258 \mathrm{~min}$ for $\mathrm{N}$-(3fluorophenyl)acetamide, $t=21.296 \mathrm{~min}$ for triphenylamine.]

\section{$N$-ethyl-4-methoxyaniline (2k) $)^{14}$}


<smiles>CCNc1ccc(OC)cc1</smiles>

Colorless oil, $13.4 \mathrm{mg}(89 \%),{ }^{1} \mathrm{H}$ NMR $\left(400 \mathrm{MHz}, \mathrm{CDCl}_{3}\right) \delta 6.83-6.74(\mathrm{~m}$, $2 \mathrm{H}), 6.63-6.56(\mathrm{~m}, 2 \mathrm{H}), 3.75(\mathrm{~s}, 3 \mathrm{H}), 3.12(\mathrm{q}, J=7.1 \mathrm{~Hz}, 2 \mathrm{H}), 1.25(\mathrm{t}, J=7.1$ $\mathrm{Hz}, 3 \mathrm{H}) .{ }^{13} \mathrm{C} \mathrm{NMR}\left(100 \mathrm{MHz}, \mathrm{CDCl}_{3}\right) \delta 152.0,142.8,114.9,114.1,55.8,39.5$, 15.0. GC conditions [Agilent DB-35, $30 \mathrm{~m} \times 0.32 \mathrm{~mm} \times 0.25 \mathrm{~mm}$, injector temp. $230{ }^{\circ} \mathrm{C}$, detector temp. $250{ }^{\circ} \mathrm{C}, 50{ }^{\circ} \mathrm{C}$ ramps $10{ }^{\circ} \mathrm{C} / \mathrm{min}$ to $300{ }^{\circ} \mathrm{C}$, hold $10 \mathrm{~min}, \mathrm{~N}_{2}$ fluency 1.0 $\mathrm{mL} / \mathrm{min} . t=11.939 \mathrm{~min}$ for 4-methoxyaniline, $t=13.475 \mathrm{~min}$ for 4-methoxy- $N$-ethylaniline, $t=$ $17.489 \mathrm{~min}$ for $N$-(4-methoxyphenyl)acetamide, $t=21.282 \mathrm{~min}$ for triphenylamine.]

\section{2-(ethylamino)phenol (2I) ${ }^{17}$}<smiles>CCNc1ccccc1O</smiles>

Dark-red solid, $11.0 \mathrm{mg}(80 \%),{ }^{1} \mathrm{H}$ NMR $\left(400 \mathrm{MHz}, \mathrm{CDCl}_{3}\right) \delta 6.87(\mathrm{td}, J=7.8,1.4$ $\mathrm{Hz}, 1 \mathrm{H}), 6.72-6.69(\mathrm{~m}, 2 \mathrm{H}), 6.63(\mathrm{td}, J=7.6,1.5 \mathrm{~Hz}, 1 \mathrm{H}), 4.44(\mathrm{bs}, 1 \mathrm{H}), 3.17$ (q, $J=$ $7.1 \mathrm{~Hz}, 2 \mathrm{H}), 1.29(\mathrm{t}, J=7.1 \mathrm{~Hz}, 3 \mathrm{H}) .{ }^{13} \mathrm{C} \mathrm{NMR}\left(100 \mathrm{MHz}, \mathrm{CDCl}_{3}\right) \delta 143.7,137.2$, $121.7,117.8,114.3,112.7,38.7,14.8$.

\section{$N$-ethylnaphthalen-1-amine (2m) $)^{10}$}

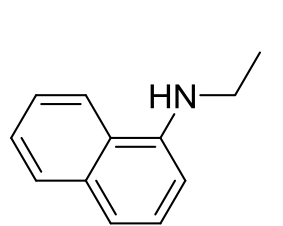

Yellow oil, $14.4 \mathrm{mg}(84 \%),{ }^{1} \mathrm{H}$ NMR $\left(400 \mathrm{MHz}, \mathrm{CDCl}_{3}\right) \delta$ 7.85-7.77 (m, 2H), 7.48$7.42(\mathrm{~m}, 2 \mathrm{H}), 7.35(\mathrm{t}, J=7.9 \mathrm{~Hz}, 1 \mathrm{H}), 7.24-7.22(\mathrm{~m}, 1 \mathrm{H}), 6.61(\mathrm{~d}, J=7.5 \mathrm{~Hz}, 1 \mathrm{H})$, 4.23 (br s, $1 \mathrm{H}), 3.31$ (q, $J=7.1 \mathrm{~Hz}, 2 \mathrm{H}), 1.40$ (t, $J=7.1 \mathrm{~Hz}, 3 \mathrm{H}) .{ }^{13} \mathrm{C}$ NMR $(100$ $\left.\mathrm{MHz}, \mathrm{CDCl}_{3}\right) \delta 143.6,134.3,128.7,126.7,125.7,124.6,123.3,119.8,117.2,104.3$, 38.7, 14.8. GC conditions [Agilent DB-35, $30 \mathrm{~m} \times 0.32 \mathrm{~mm} \times 0.25 \mathrm{~mm}$, injector temp. $230{ }^{\circ} \mathrm{C}$, detector temp. $250{ }^{\circ} \mathrm{C}, 50{ }^{\circ} \mathrm{C}$ ramps $10{ }^{\circ} \mathrm{C} / \mathrm{min}$ to $300{ }^{\circ} \mathrm{C}$, hold $10 \mathrm{~min}, \mathrm{~N}_{2}$ fluency $1.0 \mathrm{~mL} / \mathrm{min}$. $t=16.442 \mathrm{~min}$ for naphthalen-1-amine, $t=17.421 \mathrm{~min}$ for $N$-ethylnaphthalen-1-amine, $t=20.222 \mathrm{~min}$ for acetanil, $t=21.272 \mathrm{~min}$ for triphenylamine.]

\section{$\mathrm{N}$-ethyl-6-methylpyridin-2-amine (2n) ${ }^{18}$}<smiles>CCNc1cccc(C)n1</smiles>

Yellow oil, $12.4 \mathrm{mg}(91 \%),{ }^{1} \mathrm{H}$ NMR $\left(400 \mathrm{MHz}, \mathrm{CDCl}_{3}\right) \delta 7.23(\mathrm{t}, J=7.8 \mathrm{~Hz}, 1 \mathrm{H})$, $6.43(\mathrm{~d}, J=7.2 \mathrm{~Hz}, 1 \mathrm{H}), 6.18(\mathrm{~d}, J=8.2 \mathrm{~Hz}, 1 \mathrm{H}), 4.42(\mathrm{br} \mathrm{s}, 1 \mathrm{H}), 3.25-3.22(\mathrm{~m}$, $2 \mathrm{H}), 2.36(\mathrm{~s}, 3 \mathrm{H}), 1.24(\mathrm{t}, J=7.2 \mathrm{~Hz}, 3 \mathrm{H}) .{ }^{13} \mathrm{C} \mathrm{NMR}\left(100 \mathrm{MHz}, \mathrm{CDCl}_{3}\right) \delta 158.6$, 157.0, 137.9, 112.1, 102.3, 37.1, 24.3, 14.8. GC conditions [Agilent DB-35, $30 \mathrm{~m}$ $\times 0.32 \mathrm{~mm} \times 0.25 \mathrm{~mm}$, injector temp. $230{ }^{\circ} \mathrm{C}$, detector temp. $250{ }^{\circ} \mathrm{C}, 50{ }^{\circ} \mathrm{C}$ ramps $10{ }^{\circ} \mathrm{C} / \mathrm{min}$ to $300{ }^{\circ} \mathrm{C}$, hold $10 \mathrm{~min}, \mathrm{~N}_{2}$ fluency $1.0 \mathrm{~mL} / \mathrm{min} . t=9.459 \mathrm{~min}$ for 6-methylpyridin-2-amine, $t=10.944$ $\min$ for $N$-ethyl-6-methylpyridin-2-amine, $t=13.898 \mathrm{~min}$ for N-ethyl-6-methylpyridin-2-amine, $t=$ $21.284 \mathrm{~min}$ for triphenylamine.]

\section{$N$-methylaniline (2o) ${ }^{19}$}

$$
\begin{aligned}
& \text { Colorless oil, } 9.0 \mathrm{mg}(85 \%),{ }^{1} \mathrm{H} \mathrm{NMR}\left(400 \mathrm{MHz}, \mathrm{CDCl}_{3}\right) \delta 7.19(\mathrm{t}, J=7.9 \mathrm{~Hz}, 2 \mathrm{H}), 6.71 \\
& \left(100 \mathrm{MHz}, \mathrm{CDCl}_{3}\right) \delta 149.4,129.2,117.3,112.4,30.8 . \mathrm{GC} \text { conditions [Agilent DB-35, } 30 \\
& \mathrm{~m} \times 0.32 \mathrm{~mm} \times 0.25 \mathrm{~mm} \text {, injector temp. } 230{ }^{\circ} \mathrm{C} \text {, detector temp. } 250{ }^{\circ} \mathrm{C}, 50{ }^{\circ} \mathrm{C} \text { ramps }
\end{aligned}
$$

\section{$N$-propylaniline (2p) ${ }^{14}$}




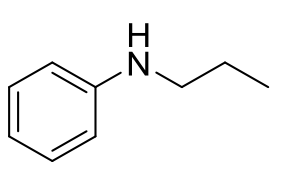

Colorless oil, $12.8 \mathrm{mg}(95 \%),{ }^{1} \mathrm{H}$ NMR $\left(400 \mathrm{MHz}^{\mathrm{C}} \mathrm{CDCl}_{3}\right) \delta 7.19(\mathrm{t}, J=7.9 \mathrm{~Hz}$, $2 \mathrm{H}), 6.70(\mathrm{t}, J=7.3 \mathrm{~Hz}, 1 \mathrm{H}), 6.65-6.60(\mathrm{~m}, J=8.6,2 \mathrm{H}), 3.09(\mathrm{t}, J=7.1 \mathrm{~Hz}, 2 \mathrm{H})$, $1.70-1.59(\mathrm{~m}, 2 \mathrm{H}), 1.01(\mathrm{t}, J=7.4 \mathrm{~Hz}, 3 \mathrm{H}) .{ }^{13} \mathrm{C} \mathrm{NMR}\left(100 \mathrm{MHz}, \mathrm{CDCl}_{3}\right) \delta 148.5$, 129.2, 117.1, 112.7, 45.8, 22.8, 11.7. GC conditions [Agilent DB-35, $30 \mathrm{~m} \times 0.32$ $\mathrm{mm} \times 0.25 \mathrm{~mm}$, injector temp. $230{ }^{\circ} \mathrm{C}$, detector temp. $250{ }^{\circ} \mathrm{C}, 50{ }^{\circ} \mathrm{C}$ ramps $10{ }^{\circ} \mathrm{C} / \mathrm{min}$ to $300{ }^{\circ} \mathrm{C}$, hold $10 \mathrm{~min}, \mathrm{~N}_{2}$ fluency $1.0 \mathrm{~mL} / \mathrm{min}$. $t=8.130 \mathrm{~min}$ for aniline, $t=11.274 \mathrm{~min}$ for $N$-propylaniline, $t=15.280 \mathrm{~min}$ for $\mathrm{N}$-phenylpropionamide, $t=21.277 \mathrm{~min}$ for triphenylamine.]

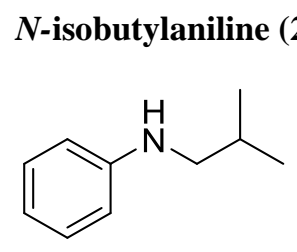

Colorless oil, $10.4 \mathrm{mg}(70 \%),{ }^{1} \mathrm{H}$ NMR $\left(400 \mathrm{MHz}, \mathrm{CDCl}_{3}\right) \delta$ 7.20-7.11 (m, $\left.2 \mathrm{H}\right)$, $6.67(\mathrm{t}, J=7.3 \mathrm{~Hz}, 1 \mathrm{H}), 6.60(\mathrm{~d}, J=7.7 \mathrm{~Hz}, 2 \mathrm{H}), 3.69(\mathrm{br} \mathrm{s}, 1 \mathrm{H}), 2.93(\mathrm{~d}, J=6.8$ $\mathrm{Hz}, 2 \mathrm{H}), 1.96-1.81(\mathrm{~m}, 1 \mathrm{H}), 0.98(\mathrm{~d}, J=6.7 \mathrm{~Hz}, 6 \mathrm{H}) .{ }^{13} \mathrm{C} \mathrm{NMR}\left(100 \mathrm{MHz}, \mathrm{CDCl}_{3}\right)$ $\delta$ 148.6, 129.2, 117.0, 112.6, 51.8, 28.0, 20.5. GC conditions [Agilent DB-35, 30 $\mathrm{m} \times 0.32 \mathrm{~mm} \times 0.25 \mathrm{~mm}$, injector temp. $230{ }^{\circ} \mathrm{C}$, detector temp. $250{ }^{\circ} \mathrm{C}, 50{ }^{\circ} \mathrm{C}$ ramps $10{ }^{\circ} \mathrm{C} / \mathrm{min}$ to $300{ }^{\circ} \mathrm{C}$, hold $10 \mathrm{~min}, \mathrm{~N}_{2}$ fluency $1.0 \mathrm{~mL} / \mathrm{min} . t=8.153 \mathrm{~min}$ for aniline, $t=11.790 \mathrm{~min}$ for $\mathrm{N}$ isobutylaniline, $t=15.061 \mathrm{~min}$ for $\mathrm{N}$-phenylisobutyramide, $t=21.273 \mathrm{~min}$ for triphenylamine.]

\section{$N$-dodecylaniline $(2 \mathbf{r})^{21}$}

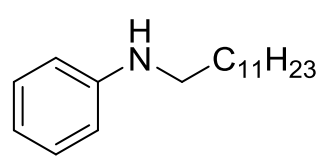

White solid, $25.0 \mathrm{mg}(96 \%),{ }^{1} \mathrm{H}$ NMR $\left(400 \mathrm{MHz}, \mathrm{CDCl}_{3}\right) \delta$ 7.20-7.13 (m, $\left.2 \mathrm{H}\right)$, $6.68(\mathrm{t}, J=7.3 \mathrm{~Hz}, 1 \mathrm{H}), 6.64-6.56(\mathrm{~m}, 2 \mathrm{H}), 3.59(\mathrm{br} \mathrm{s}, 1 \mathrm{H}), 3.10(\mathrm{t}, J=7.1 \mathrm{~Hz}$, $2 \mathrm{H}), 1.67-1.56(\mathrm{~m}, 2 \mathrm{H}), 1.44-1.17(\mathrm{~m}, 18 \mathrm{H}), 0.88(\mathrm{t}, J=6.8 \mathrm{~Hz}, 3 \mathrm{H}) .{ }^{13} \mathrm{C} \mathrm{NMR}$ $\left(100 \mathrm{MHz}, \mathrm{CDCl}_{3}\right) \delta 148.6,129.2,117.1,112.7,44.0,31.9,29.7,29.66,29.63$, 29.7, 29.5, 29.4, 27.2, 22.7, 14.2. GC conditions [Agilent DB-35, $30 \mathrm{~m} \times 0.32$ $\mathrm{mm} \times 0.25 \mathrm{~mm}$, injector temp. $230{ }^{\circ} \mathrm{C}$, detector temp. $250{ }^{\circ} \mathrm{C}, 50{ }^{\circ} \mathrm{C}$ ramps $10^{\circ} \mathrm{C} / \mathrm{min}$ to $300{ }^{\circ} \mathrm{C}$, hold $10 \mathrm{~min}, \mathrm{~N}_{2}$ fluency $1.0 \mathrm{~mL} / \mathrm{min} . t=8.156 \mathrm{~min}$ for aniline, $t=21.038 \mathrm{~min}$ for $N$-dodecylaniline, $t=22.463 \mathrm{~min}$ for $N$-phenyldodecanamide, $t=21.290 \mathrm{~min}$ for triphenylamine.]

\section{$N$-(2-(benzyloxy)ethyl)aniline (2s) $)^{22}$}<smiles>c1ccc(CCCOCc2ccccc2)cc1</smiles>

White solid, $18.8 \mathrm{mg}(83 \%),{ }^{1} \mathrm{H}$ NMR $\left(400 \mathrm{MHz}, \mathrm{CDCl}_{3}\right) \delta$ 7.40-7.28 (m, 5H), 7.22-7.14 (m, 2H), 6.76-6.60 (m, 3H), $4.56(\mathrm{~s}, 2 \mathrm{H}), 4.06$ (br s, $1 \mathrm{H}), 3.71$ (t, $J$ $=5.3 \mathrm{~Hz}, 2 \mathrm{H}), 3.37-3.30(\mathrm{~m}, 2 \mathrm{H}) .{ }^{13} \mathrm{C} \mathrm{NMR}\left(100 \mathrm{MHz}, \mathrm{CDCl}_{3}\right) \delta 148.2,138.0$, 129.2, 128.5, 127.81, 127.78, 117.6, 113.2, 73.1, 68.7, 43.6. GC conditions [Agilent DB-35, $30 \mathrm{~m} \times 0.32 \mathrm{~mm} \times 0.25 \mathrm{~mm}$, injector temp. $230{ }^{\circ} \mathrm{C}$, detector temp. $250{ }^{\circ} \mathrm{C}, 50{ }^{\circ} \mathrm{C}$ ramps $10{ }^{\circ} \mathrm{C} / \mathrm{min}$ to $300{ }^{\circ} \mathrm{C}$, hold $10 \mathrm{~min}, \mathrm{~N}_{2}$ fluency $1.0 \mathrm{~mL} / \mathrm{min} . t=8.165 \mathrm{~min}$ for aniline, $t=$ $20.972 \mathrm{~min}$ for $N$-(2-(benzyloxy)ethyl)aniline, $t=22.726 \mathrm{~min}$ for 2-(benzyloxy)- $N$-phenylacetamide, $t=21.290 \mathrm{~min}$ for triphenylamine.]

\section{$N$-(2-(thiophen-2-yl)ethyl)aniline (2t) ${ }^{23}$}<smiles>c1ccc(NCCc2cccs2)cc1</smiles>

Yellow oil, $18.0 \mathrm{mg}(88 \%),{ }^{1} \mathrm{H}$ NMR $\left(400 \mathrm{MHz}, \mathrm{CDCl}_{3}\right) \delta 7.24-7.16(\mathrm{~m}$, $3 \mathrm{H}), 6.97(\mathrm{dd}, J=5.1,3.4 \mathrm{~Hz}, 1 \mathrm{H}), 6.90-6.85(\mathrm{~m}, 1 \mathrm{H}), 6.77-6.70(\mathrm{~m}, 1 \mathrm{H})$, 6.67-6.59 (m, 2H), 3.79 (br s, 1H), $3.45(\mathrm{t}, J=6.7 \mathrm{~Hz}, 2 \mathrm{H}), 3.15$ (t, $J=6.7$ $\mathrm{Hz}, 2 \mathrm{H}) .{ }^{13} \mathrm{C}$ NMR $\left(100 \mathrm{MHz}, \mathrm{CDCl}_{3}\right) \delta 147.8,141.8,129.4,127.0,125.3$, 123.9, 117.7, 113.1, 45.2, 29.7. GC conditions [Agilent DB-35, $30 \mathrm{~m} \times 0.32 \mathrm{~mm} \times 0.25 \mathrm{~mm}$, injector temp. $230{ }^{\circ} \mathrm{C}$, detector temp. $250{ }^{\circ} \mathrm{C}, 50{ }^{\circ} \mathrm{C}$ ramps $10{ }^{\circ} \mathrm{C} / \mathrm{min}$ to $300{ }^{\circ} \mathrm{C}$, hold $10 \mathrm{~min}, \mathrm{~N}_{2}$ fluency 
$1.0 \mathrm{~mL} / \mathrm{min} . t=8.155 \mathrm{~min}$ for aniline, $t=19.163 \mathrm{~min}$ for $N$-(2-(thiophen-2-yl)ethyl)aniline, $t=$ $21.510 \mathrm{~min}$ for 2-(benzyloxy)- $N$-phenylacetamide, $t=21.276 \mathrm{~min}$ for triphenylamine.]

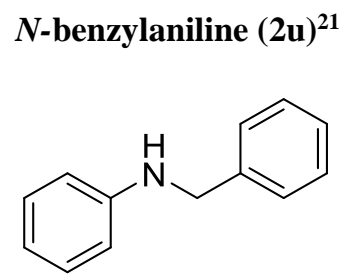

Colorless oil, $14.0 \mathrm{mg}(76 \%),{ }^{1} \mathrm{H}$ NMR $\left(400 \mathrm{MHz}, \mathrm{CDCl}_{3}\right) \delta$ 7.44-7.27 (m, 5H), $7.17(\mathrm{t}, J=7.9 \mathrm{~Hz}, 2 \mathrm{H}), 6.71(\mathrm{t}, J=7.3 \mathrm{~Hz}, 1 \mathrm{H}), 6.66(\mathrm{~d}, J=7.7 \mathrm{~Hz}, 2 \mathrm{H}), 4.32$ (s, 2H), 4.01 (br s, $1 \mathrm{H}) .{ }^{13} \mathrm{C}$ NMR $\left(100 \mathrm{MHz}, \mathrm{CDCl}_{3}\right) \delta 148.2,139.5,129.3$, 128.7, 127.6, 127.3, 117.6, 112.9, 48.3. GC conditions [Agilent DB-35, $30 \mathrm{~m} \times$ $0.32 \mathrm{~mm} \times 0.25 \mathrm{~mm}$, injector temp. $230^{\circ} \mathrm{C}$, detector temp. $250{ }^{\circ} \mathrm{C}, 50^{\circ} \mathrm{C}$ ramps $10{ }^{\circ} \mathrm{C} / \mathrm{min}$ to $300{ }^{\circ} \mathrm{C}$, hold $10 \mathrm{~min}, \mathrm{~N}_{2}$ fluency $1.0 \mathrm{~mL} / \mathrm{min} . t=8.118 \mathrm{~min}$ for aniline, $t=18.077 \mathrm{~min}$ for $N$-benzylaniline, $t=20.812 \mathrm{~min}$ for $N$-phenylbenzamide, $t=21.274 \mathrm{~min}$ for triphenylamine.]

\section{$N$-(furan-2-ylmethyl)aniline $(2 v)^{24}$}<smiles>c1ccc(NCc2ccco2)cc1</smiles>

Colorless oil, $13.8 \mathrm{mg}(85 \%),{ }^{1} \mathrm{H}$ NMR (400 MHz, $\left.\mathrm{CDCl}_{3}\right) \delta$ 7.41-7.35 (m, 1H), $7.22-7.13(\mathrm{~m}, 2 \mathrm{H}), 6.75(\mathrm{t}, J=7.3 \mathrm{~Hz}, 1 \mathrm{H}), 6.71-6.62(\mathrm{~m}, 2 \mathrm{H}), 6.36-6.21(\mathrm{~m}$, $2 \mathrm{H}), 4.31(\mathrm{~s}, 2 \mathrm{H}), 4.01$ (br s, $1 \mathrm{H}) .{ }^{13} \mathrm{C} \mathrm{NMR}\left(100 \mathrm{MHz}, \mathrm{CDCl}_{3}\right) \delta 152.8,147.6$, 142.0, 129.3, 118.0, 113.2, 110.4, 107.0, 41.5. GC conditions [Agilent DB-35, $30 \mathrm{~m} \times 0.32 \mathrm{~mm} \times 0.25 \mathrm{~mm}$, injector temp. $230{ }^{\circ} \mathrm{C}$, detector temp. $250{ }^{\circ} \mathrm{C}, 50{ }^{\circ} \mathrm{C}$ ramps $10{ }^{\circ} \mathrm{C} / \mathrm{min}$ to $300{ }^{\circ} \mathrm{C}$, hold $10 \mathrm{~min}, \mathrm{~N}_{2}$ fluency $1.0 \mathrm{~mL} / \mathrm{min}$. $t=8.129 \mathrm{~min}$ for aniline, $t=15.666 \mathrm{~min}$ for $N$ (furan-2-ylmethyl)aniline, $t=18.572 \mathrm{~min}$ for $N$-phenylfuran-2-carboxamide, $t=21.289 \mathrm{~min}$ for triphenylamine.]

\section{References}

1 Li, W.; Xie, J.-H.; Lin, H.; Zhou, Q.-L. Green Chem. 2012, 14, 2388-2390.

2 Clarke, Z. E.; Maragh, P. T.; Dasgupta, T. P.; Gusev, D. G.; Lough, A. J.; Abdur-Rashid, K. Organometallics 2006, 25, 4113-4117.

3 Liu, C.; Xie, J.-H.; Tian, G.-L.; Li, W.; Zhou, Q.-L. Chem. Sci. 2015, 6, 2928-2931.

${ }^{4}$ Rimoldi, M.; Mezzetti, A. Inorg. Chem. 2014, 53, 11974-11984.

5 Gupta, M.; Hagen, C.; Flesher, R. J.; Kaska, W. C.; Jensen, C. M. Chem. Commun. 1996, 2083-2084.

6 Göttker-Schnetmann, I.; White, P.; Brookhart, M. J. Am. Chem. Soc. 2004, 126, 1804-1811.

7 Morales-Morales, D.; Redón, R. o.; Yung, C.; Jensen, C. M. Inorg. Chim. Acta 2004, 357, 2953-2956.

8 Thomson, J. W.; Hatnean, J. A.; Hastie, J. J.; Pasternak, A.; Stephan, D. W.; Chase, P. A. Org. Process Res. \& Dev. 2013, 17, 1287-1292.

9 Song, Q.-W.; Yu, B.; Li, X.-D.; Ma, R.; Diao, Z.-F.; Li, R.-G.; Li, W.; He, L.-N. Green Chem. 2014, 16, 1633-1638.

10 Green, R. A.; Hartwig, J. F. Angew. Chem. Int. Ed. 2015, 54, 3768-3772.

11 Nacario, R.; Kotakonda, S.; Fouchard, D. M. D.; Tillekeratne, L. M. V.; Hudson, R. A. Org. Lett. 2005, 7, 471-474.

12 Stroh, R.; Ebersberger, J.; Haberland, H.; Hahn, W. Angew. Chem. 1957, 69, 124-131.

13 Sopchik, A. E.; Kingsbury, C. A. J. Chem. Soc., Perkin Trans. 2 1979, 1058-1063.

${ }^{14}$ Sorribes, I.; Cabrero-Antonino, J. R.; Vicent, C.; Junge, K.; Beller, M. J. Am. Chem. Soc. 2015, 137, 13580-13587.

15 Murali, A.; Puppala, M.; Varghese, B.; Baskaran, S. Eur. J. Org. Chem. 2011, 2011, 5297-5302. 
16 Thorstensson, F.; Kvarnström, I.; Musil, D.; Nilsson, I.; Samuelsson, B. J. Med. Chem. 2003, 46, 1165-1179.

17 Neogi, S.; Naskar, D. Syn. Comm. 2011, 41, 1901-1915.

${ }^{18}$ Green, R. A.; Hartwig, J. F. Org. Lett. 2014, 16, 4388-4391.

${ }^{19}$ Beydoun, K.; vom Stein, T.; Klankermayer, J.; Leitner, W. Angew. Chem. Inter. Ed. 2013, 52, $9554-$ 9557.

${ }^{20}$ Xiao, Q.; Tian, L.; Tan, R.; Xia, Y.; Qiu, D.; Zhang, Y.; Wang, J. Org. Lett. 2012, 14, 4230-4233.

${ }^{21}$ Zou, Q.; Wang, C.; Smith, J.; Xue, D.; Xiao, J. Chem. Eur. J. 2015, 21, 9656-9661.

${ }^{22}$ Sakai, N.; Takeoka, M.; Kumaki, T.; Asano, H.; Konakahara, T.; Ogiwara, Y. Tetrahedron Lett. 2015, 56, 6448-6451.

${ }^{23}$ Hollmann, D.; Bähn, S.; Tillack, A.; Beller, M. Angew. Chem. Inter. Ed. 2007, 46, 8291-8294.

${ }^{24}$ Sun, Y.-W.; Tang, X.-Y.; Shi, M. Chem. Commun. 2015, 51, 13937-13940. 


\section{GC and NMR Spectra of the Hydrogenation Products}

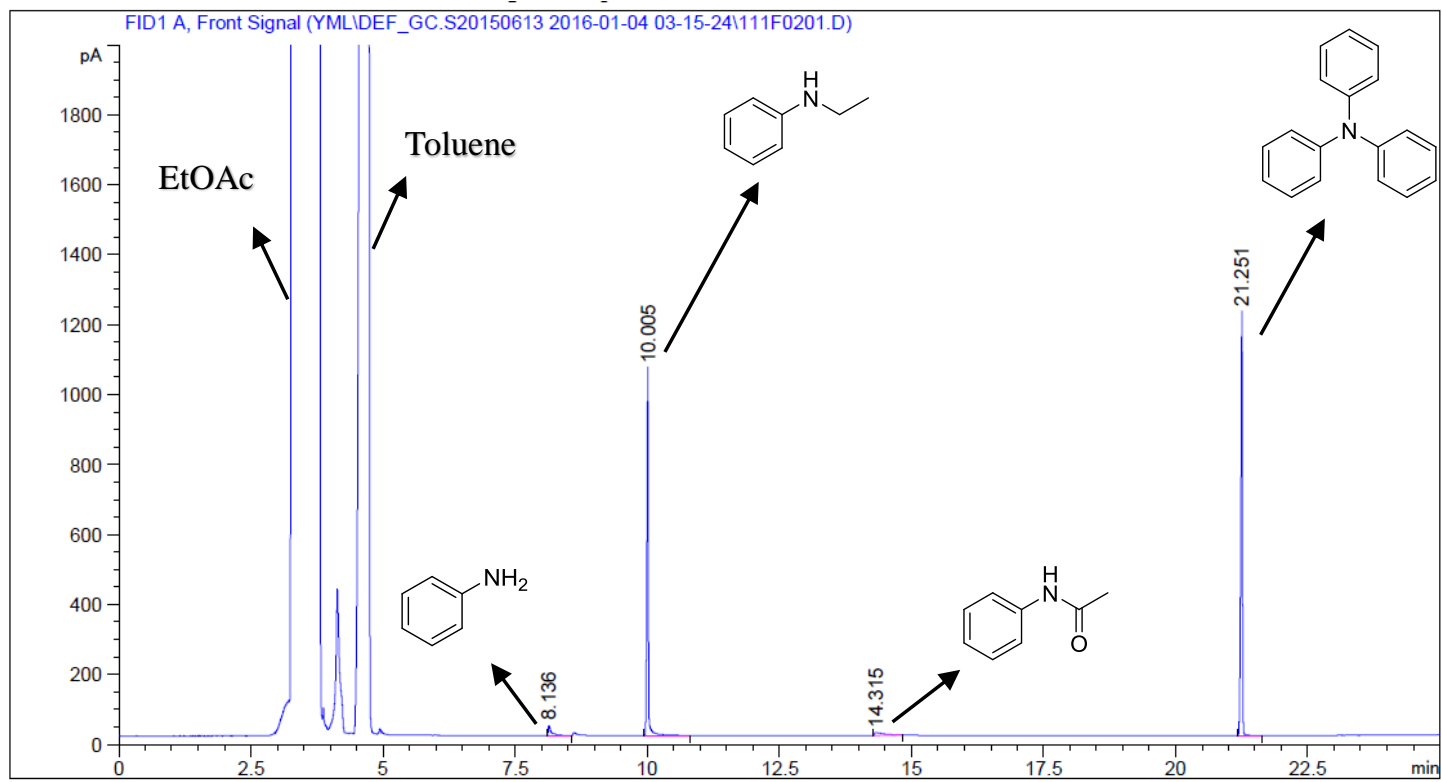

Representative chromatogram (Table $\mathrm{S} 1$, entry 2) (Temperature program: $\left.50{ }^{\circ} \mathrm{C}-10{ }^{\circ} \mathrm{C} / \mathrm{min}-300{ }^{\circ} \mathrm{C}(10 \mathrm{~min})\right)$

\begin{tabular}{|c|c|c|c|c|c|c|}
\hline $\begin{array}{c}\text { eak } \\
\#\end{array}$ & $\begin{array}{c}\text { RetTime } \\
\text { [min] }\end{array}$ & Type & $\begin{array}{l}\text { Width } \\
\text { [min] }\end{array}$ & $\begin{array}{r}\text { Area } \\
{\left[\mathrm{pA}^{\star} \mathrm{s}\right]}\end{array}$ & $\begin{array}{l}\text { Height } \\
{[\mathrm{pA}]}\end{array}$ & $\begin{array}{c}\text { Area } \\
\quad \%\end{array}$ \\
\hline & & & & & & \\
\hline 1 & 8 & $D P$ & 0.0537 & 115.86826 & 06695 & 382 \\
\hline 2 & 10 & BB & 319 & 2221.11011 & 4509 & 42 . \\
\hline 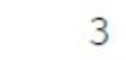 & & & & 113. & 455 & 944 \\
\hline A & 51 & & & 2760.31641 & 5825 & 52. \\
\hline
\end{tabular}


NMR Spectra of The Isolated Products

$N$-ethylaniline (2a)
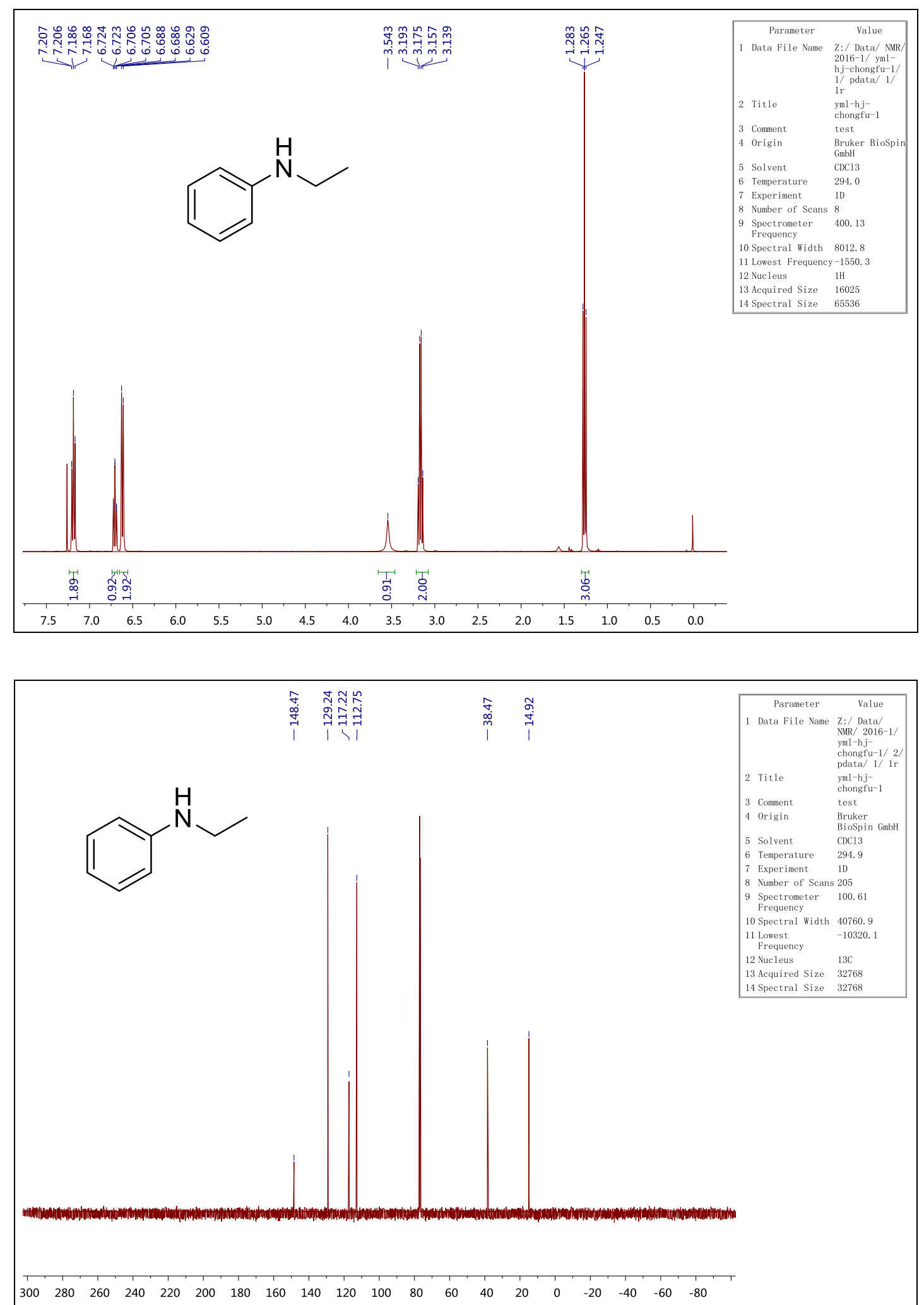
$\mathrm{N}$-ethyl-2-methylaniline (2b)
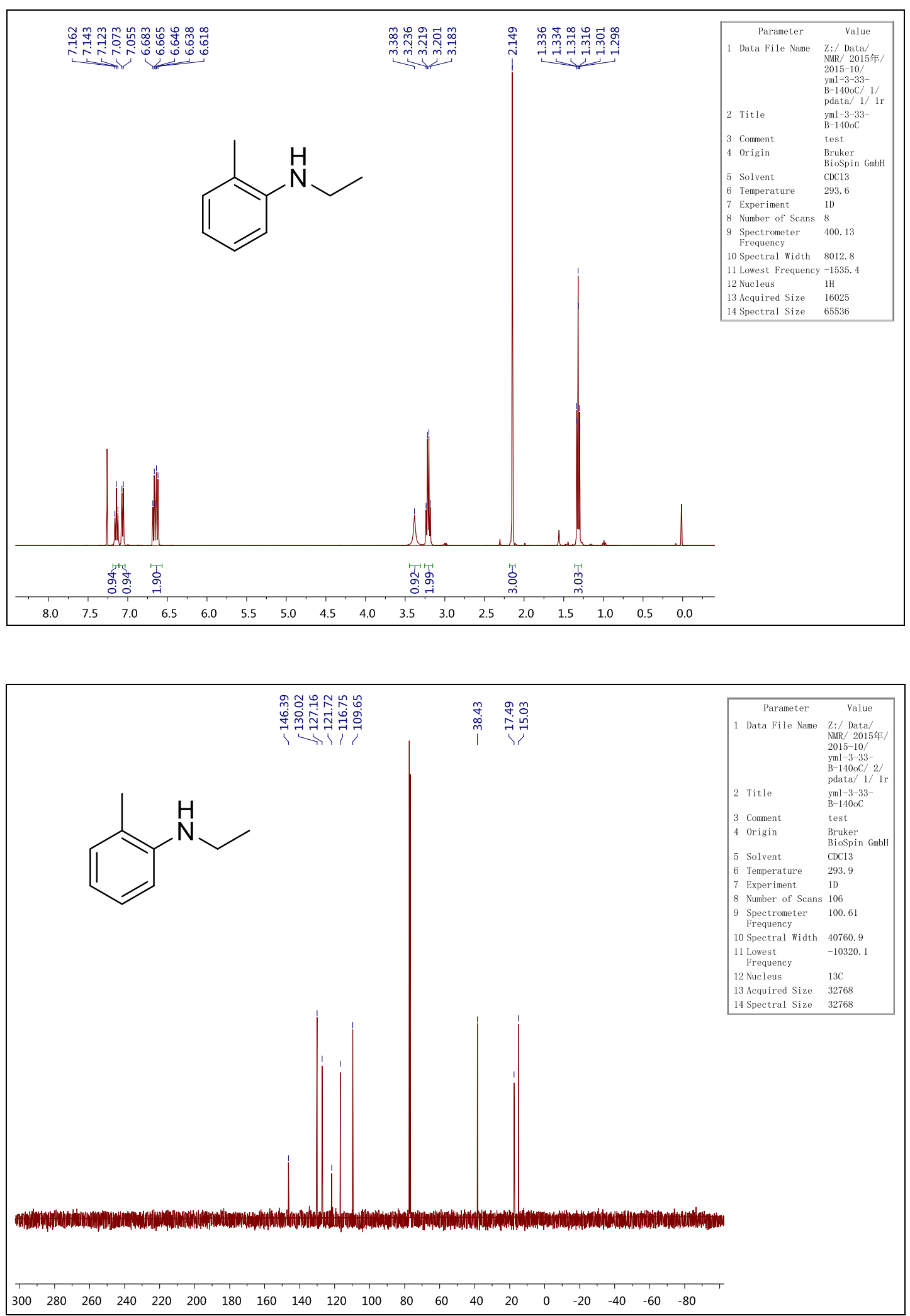
$\mathrm{N}$-ethyl-3-methylaniline (2c)
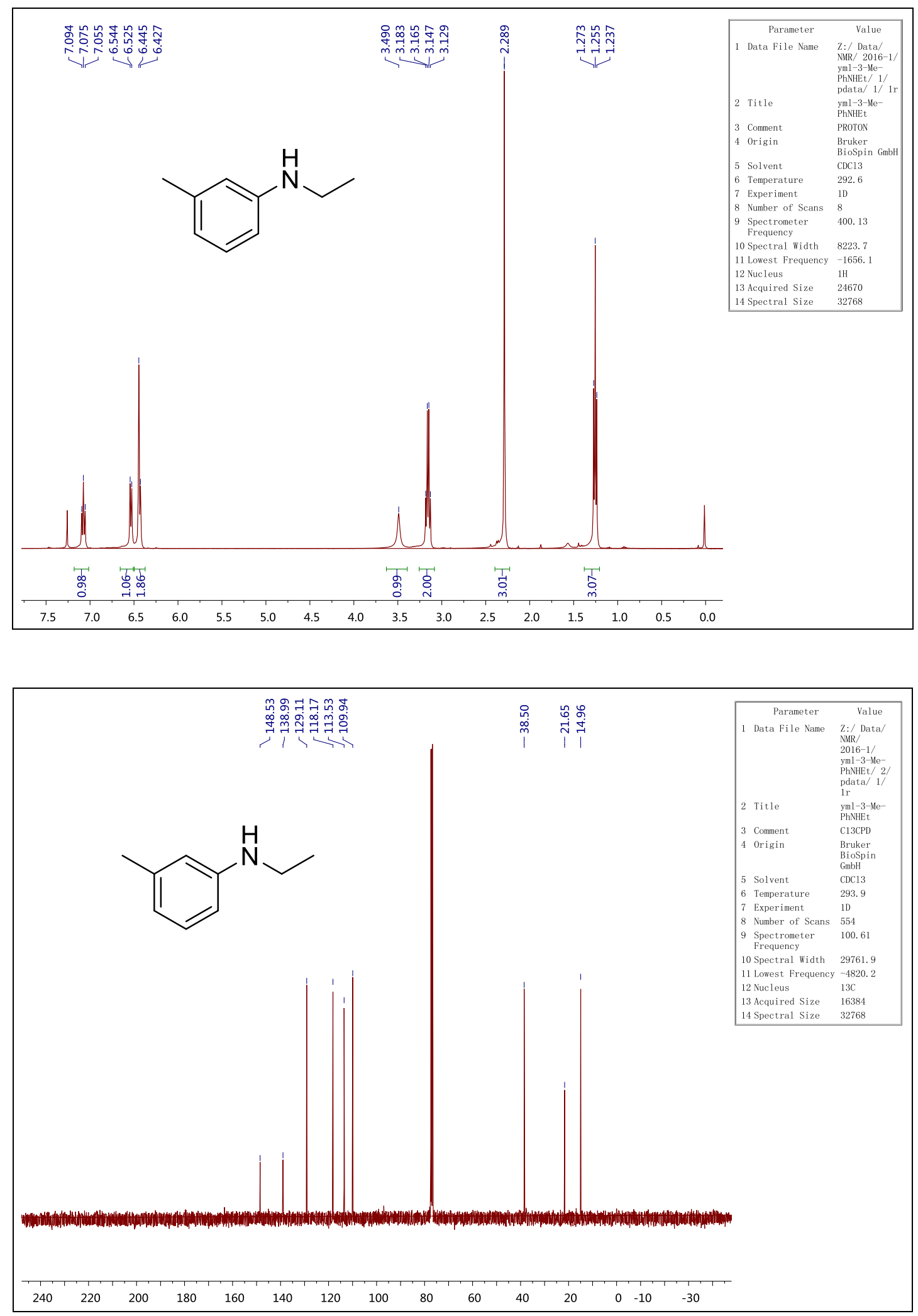
$N$-ethyl-4-methylaniline (2d)
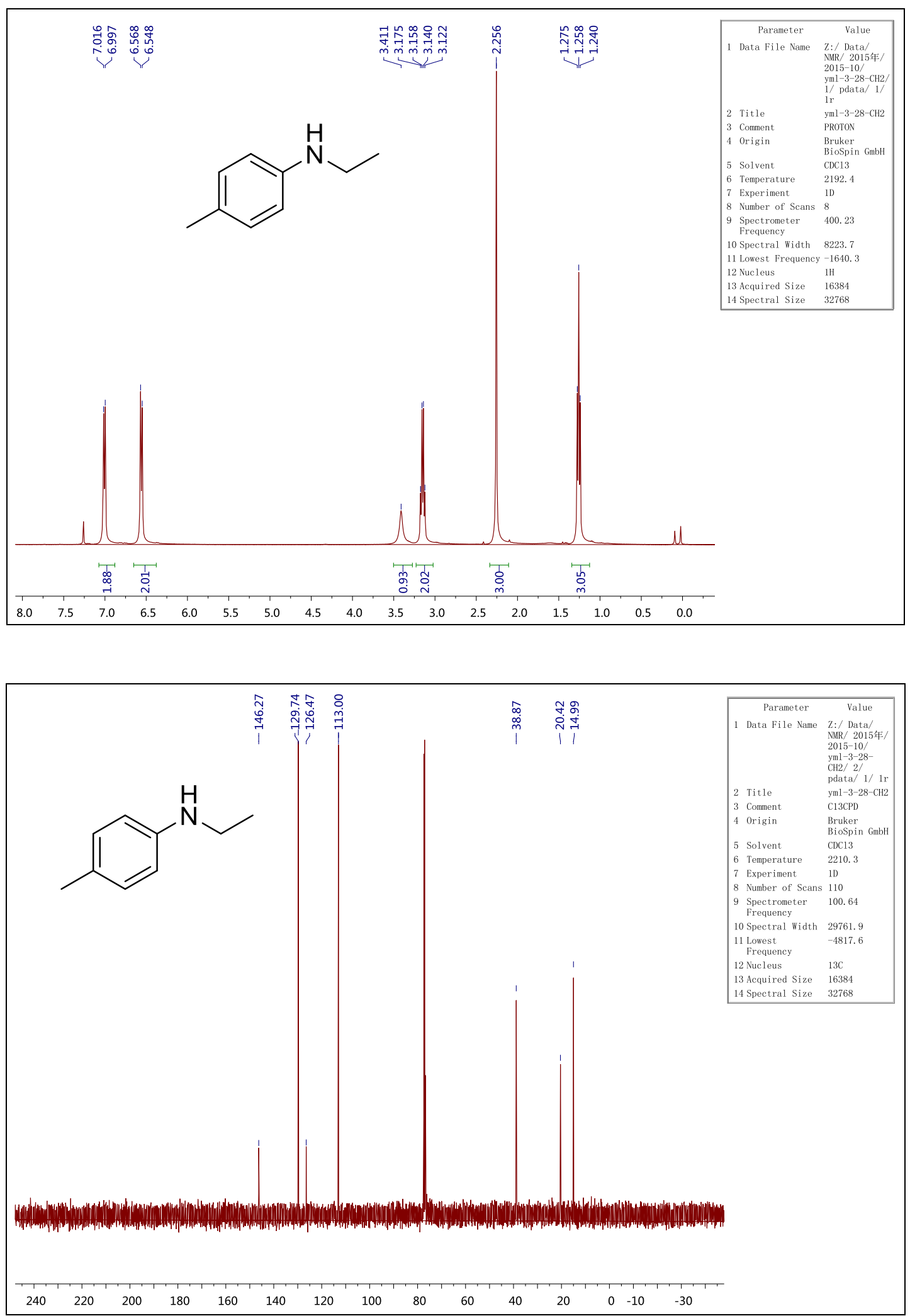
$\mathrm{N}$-ethyl-2,4-dimethylaniline (2e)
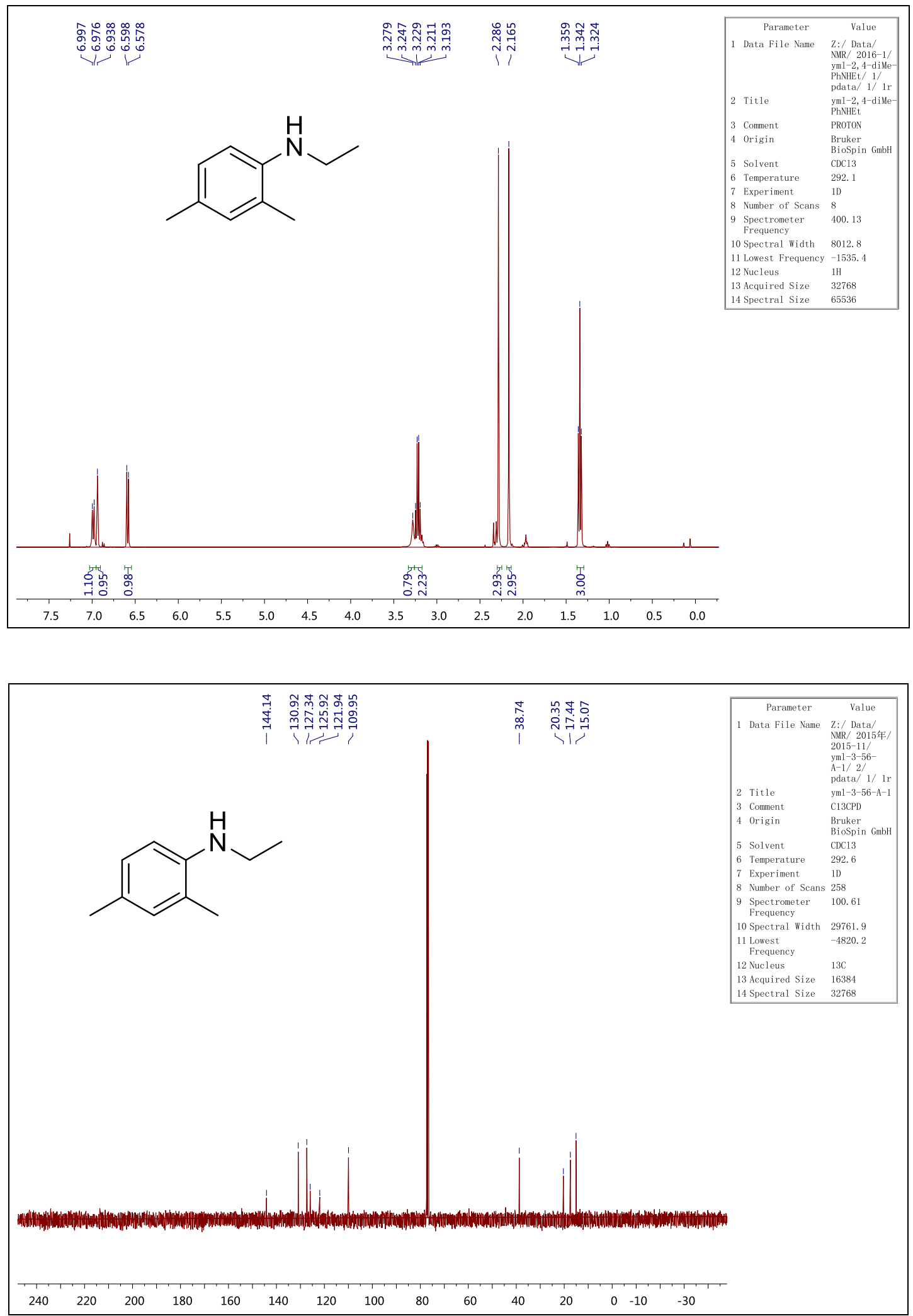
2-chloro- $N$-ethylaniline (2f)
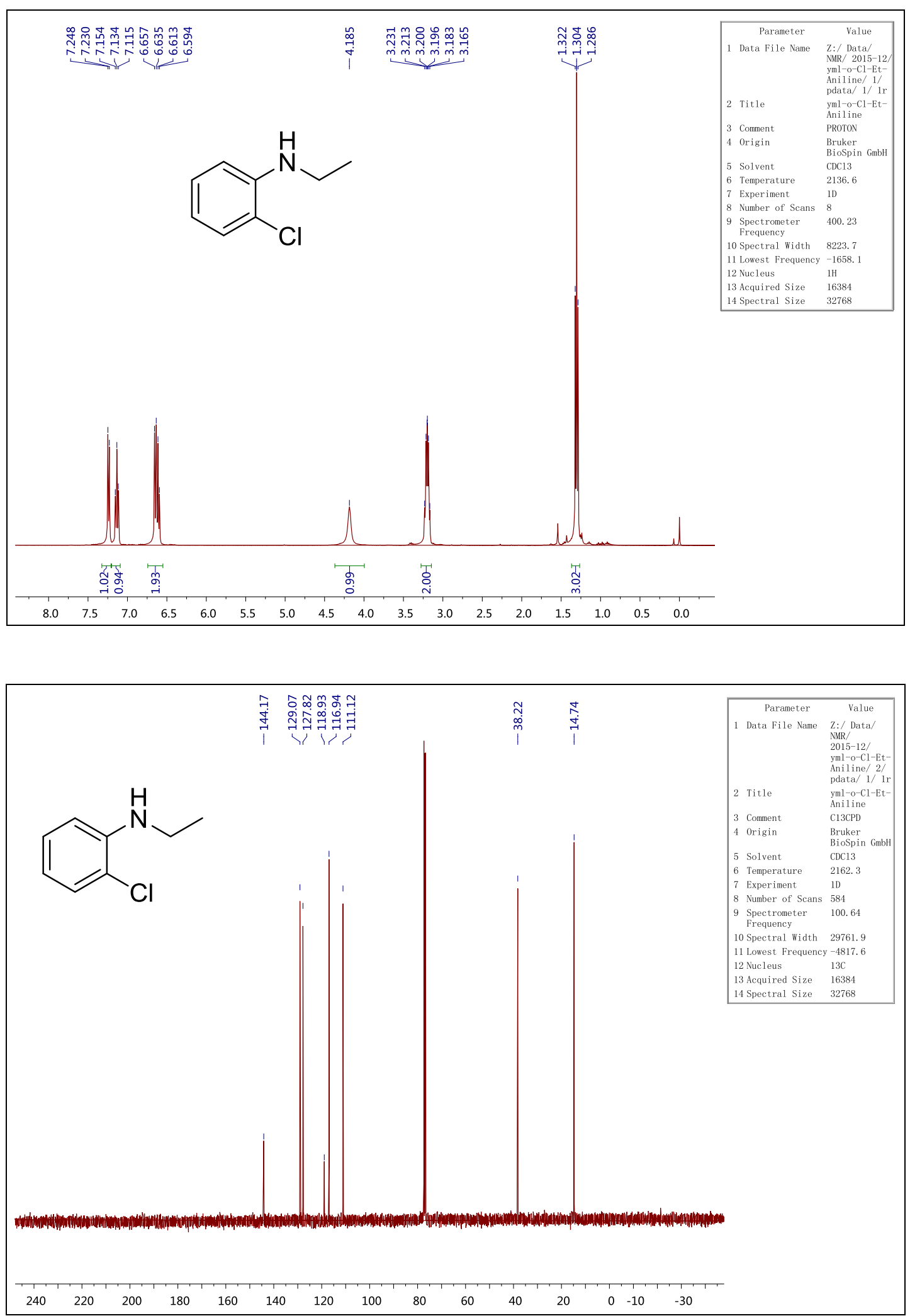
3-chloro- $N$-ethylaniline (2g)
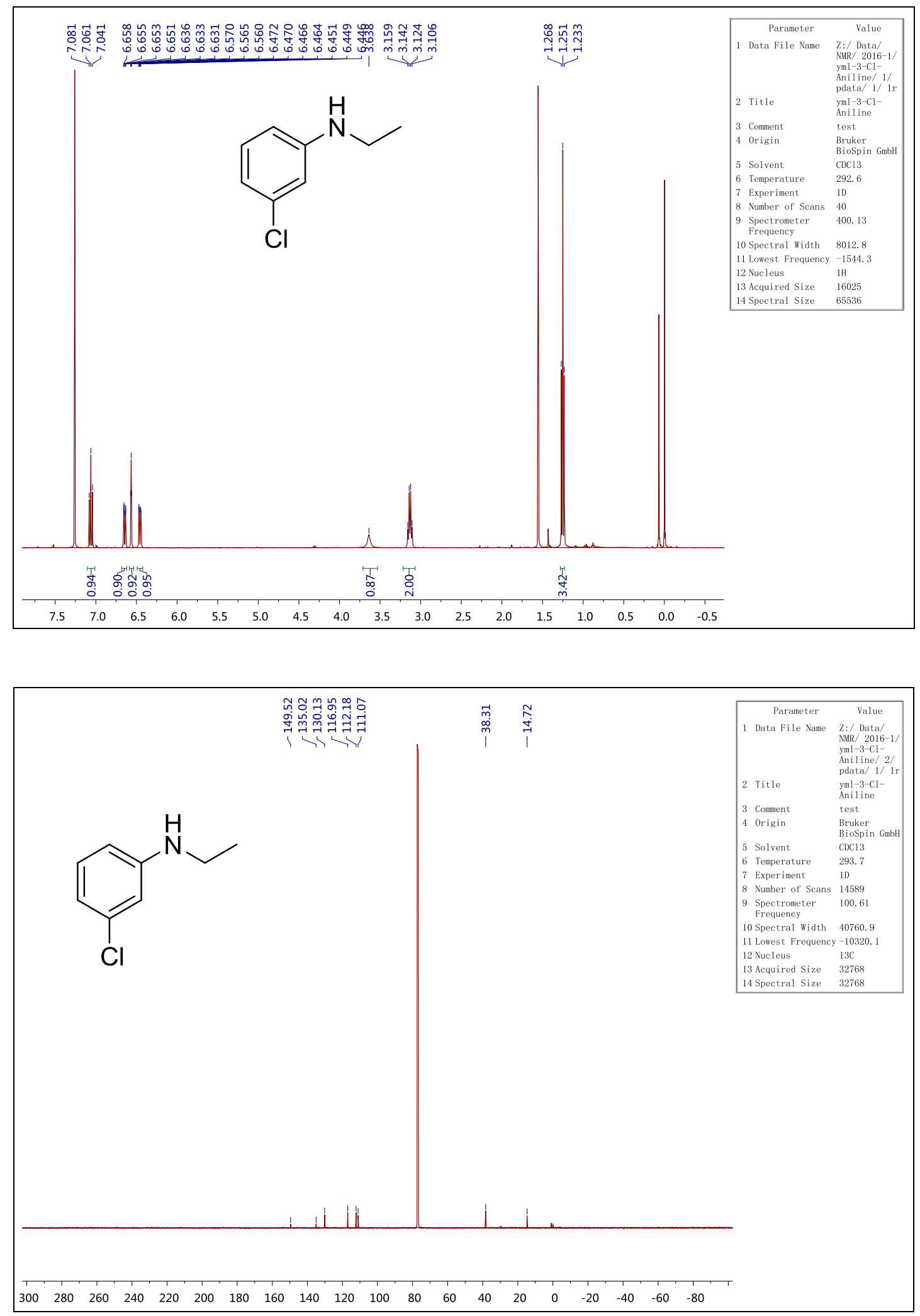
4-chloro- $N$-ethylaniline (2h)
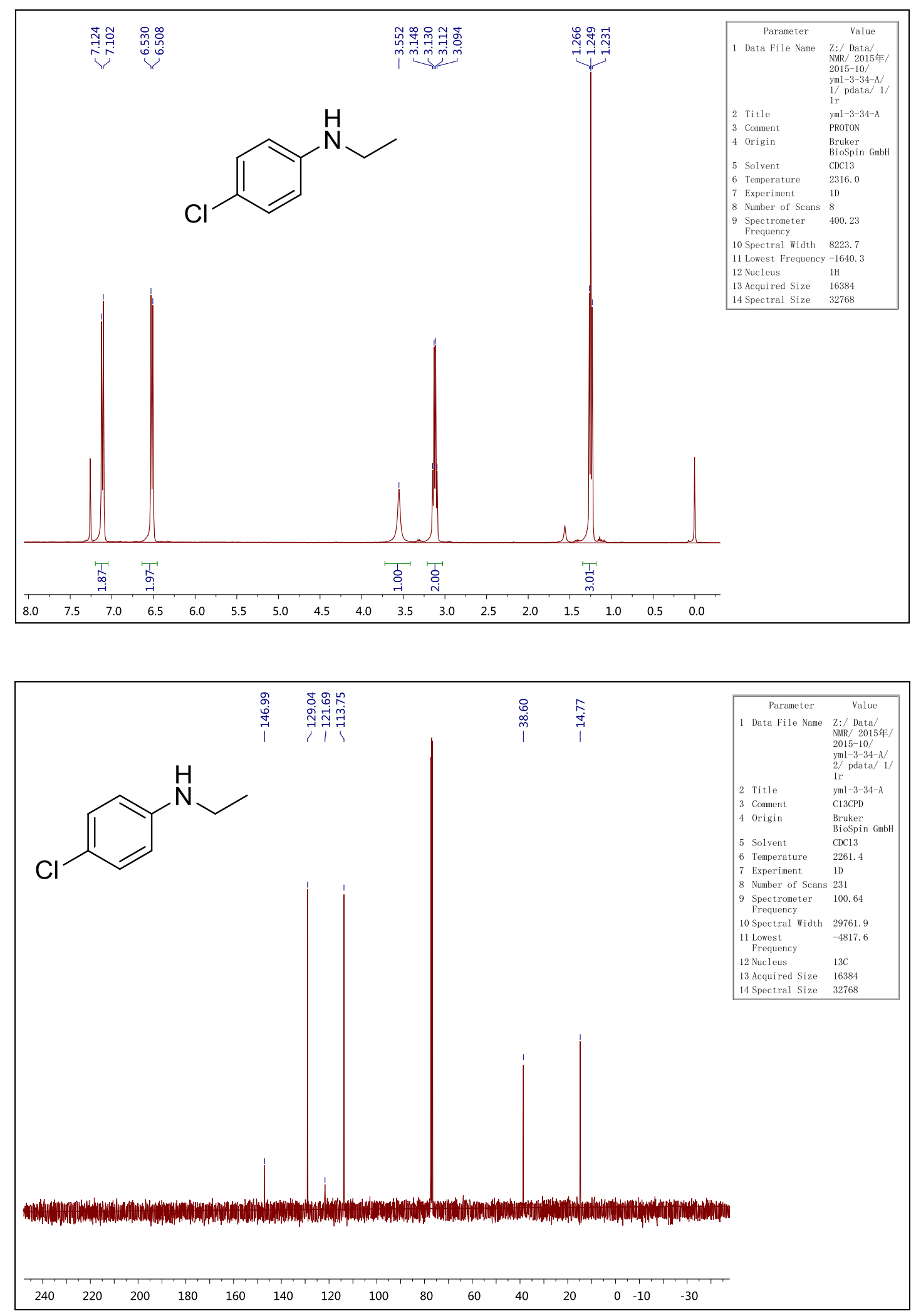
4-bromo- $N$-ethylaniline (2i)
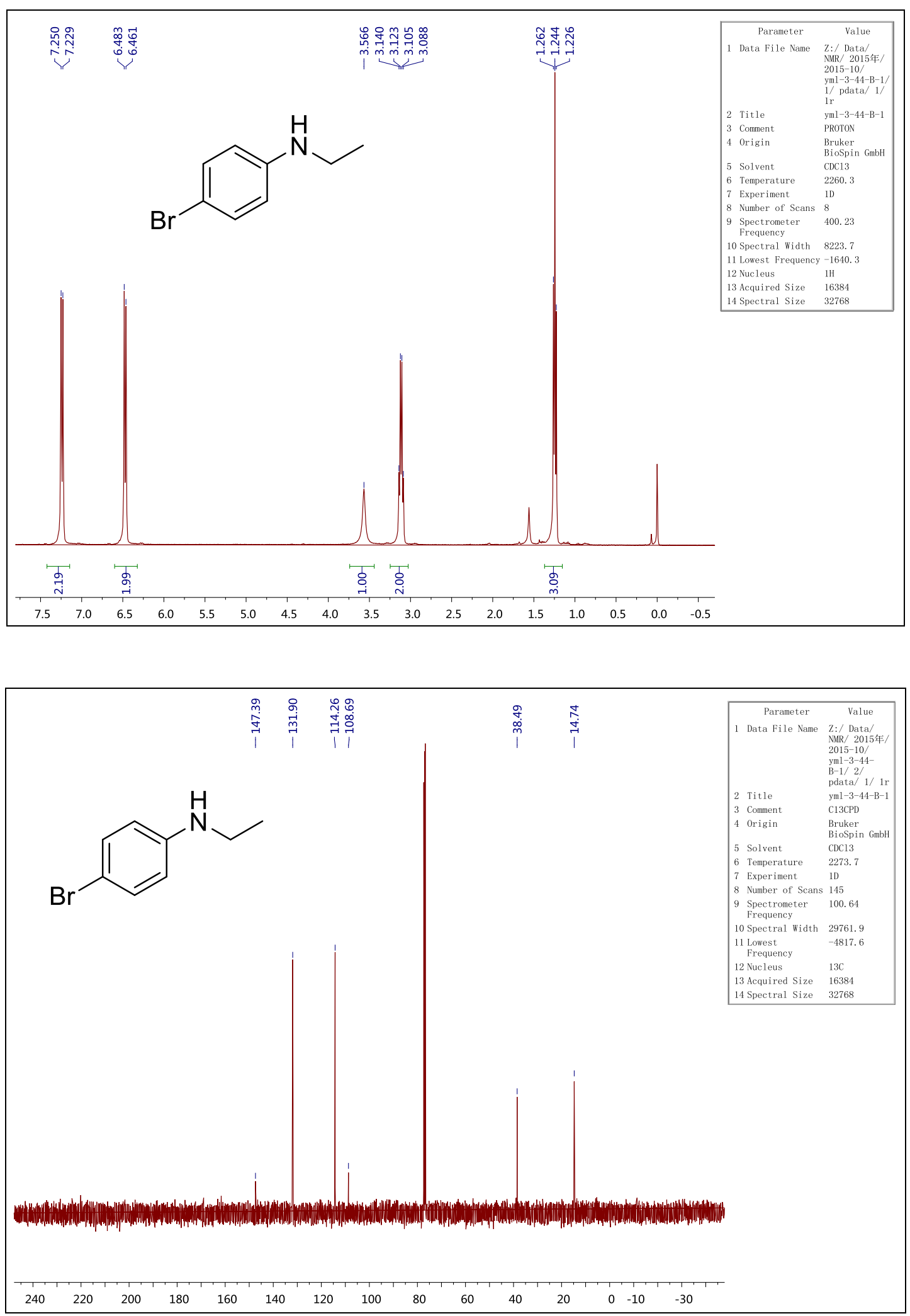
$\mathrm{N}$-ethyl-3-fluoroaniline (2j)
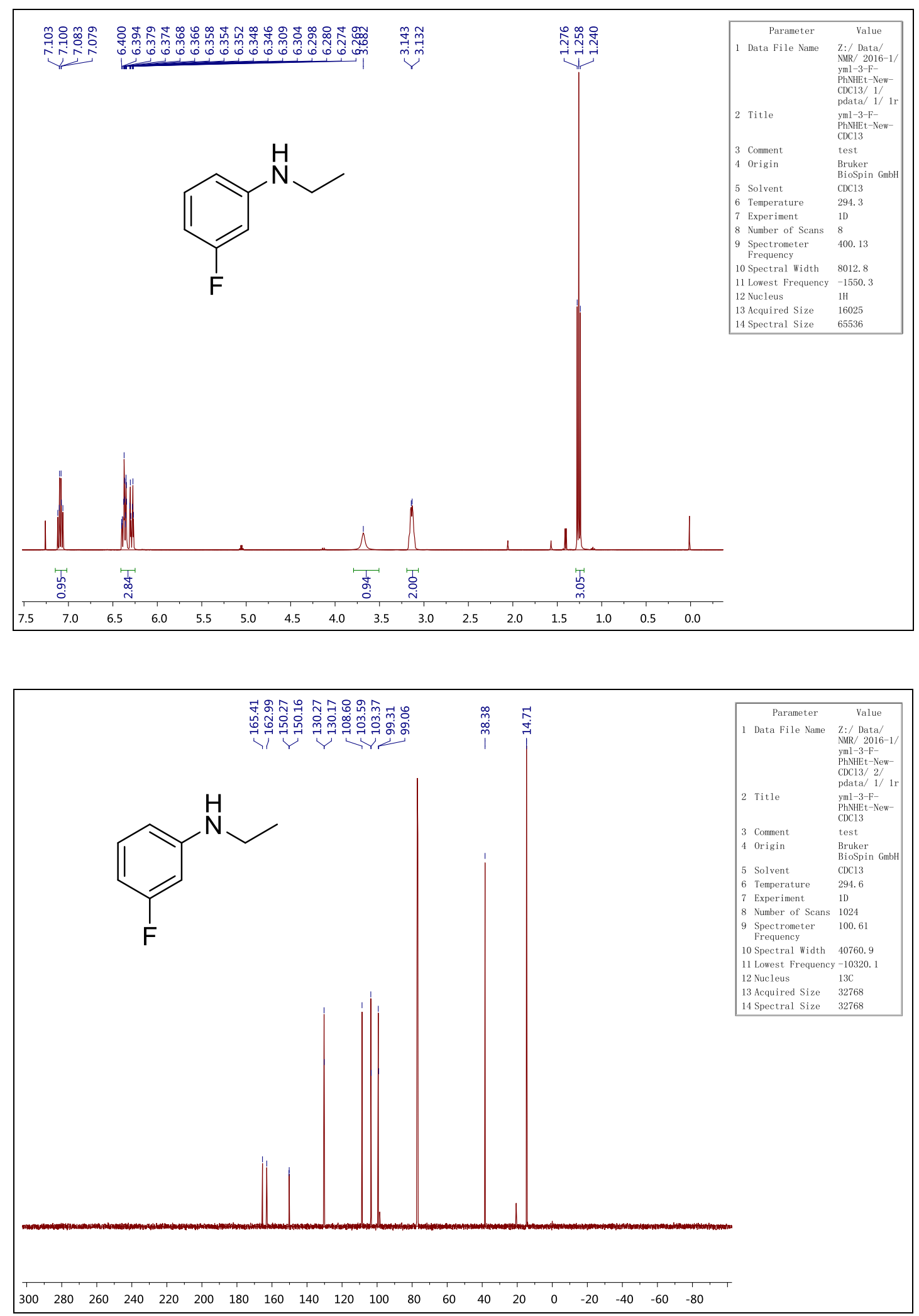
$\mathrm{N}$-ethyl-4-methoxyaniline (2k)
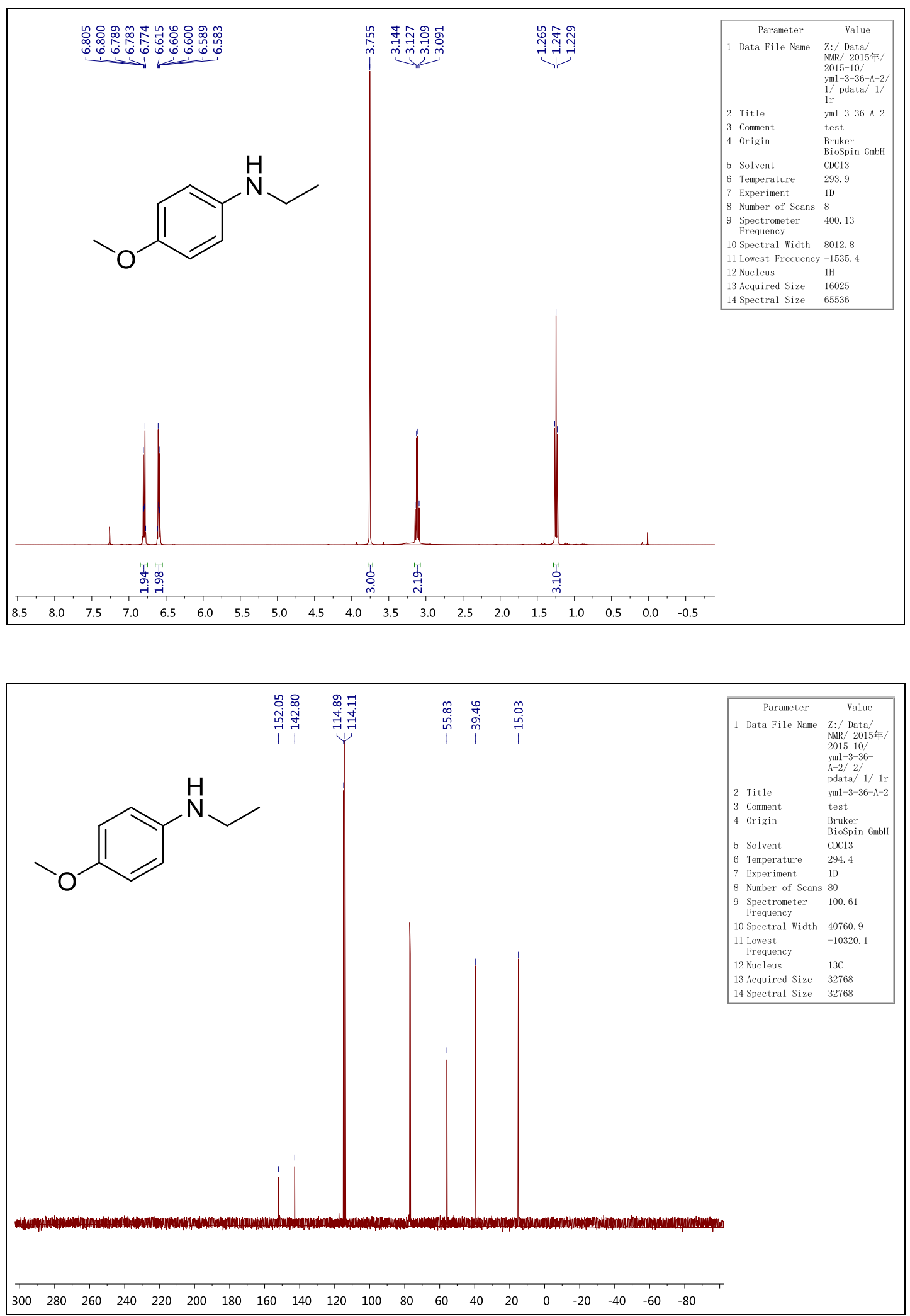
2-(ethylamino)phenol (2l)
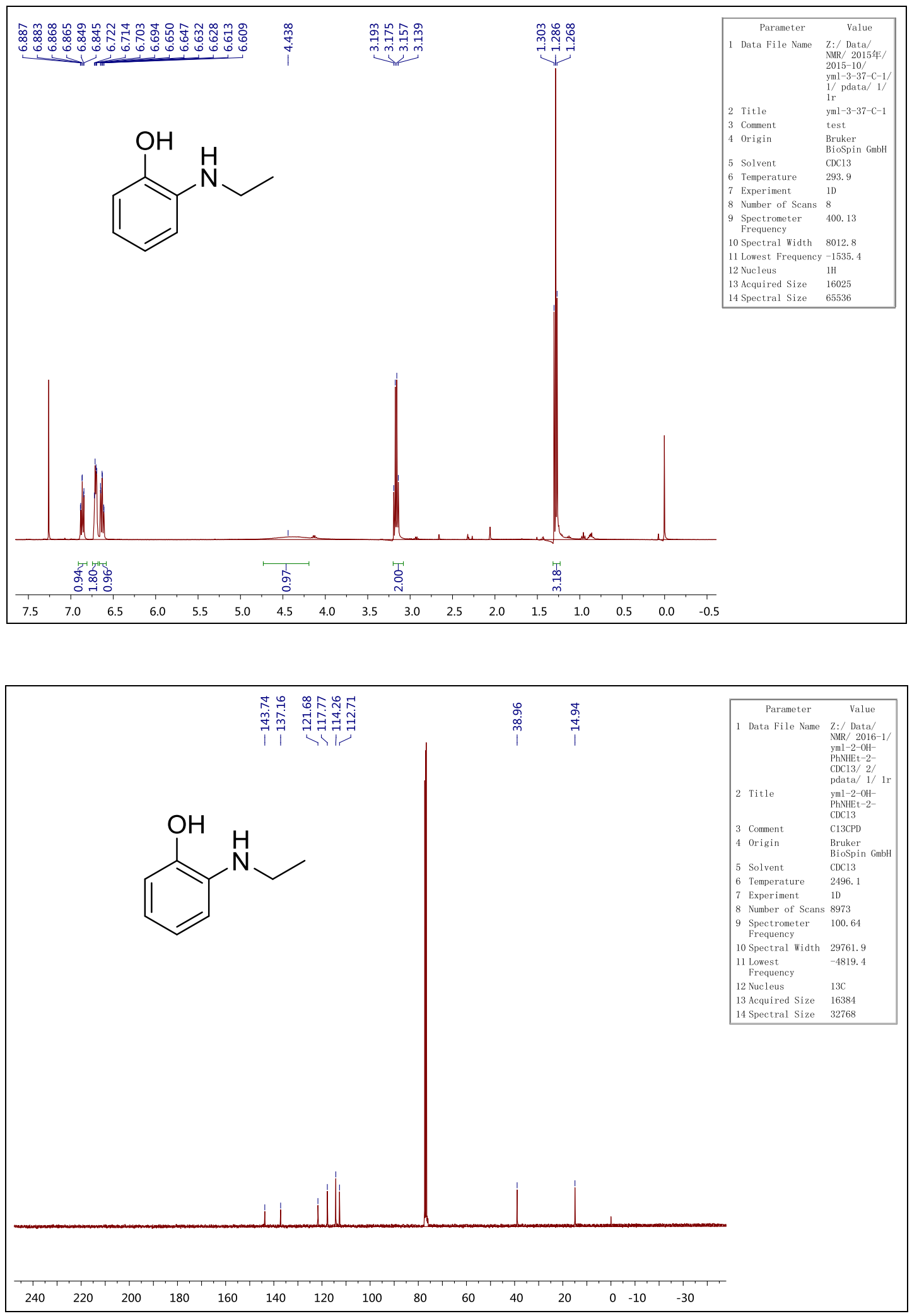
$N$-ethylnaphthalen-1-amine (2m)
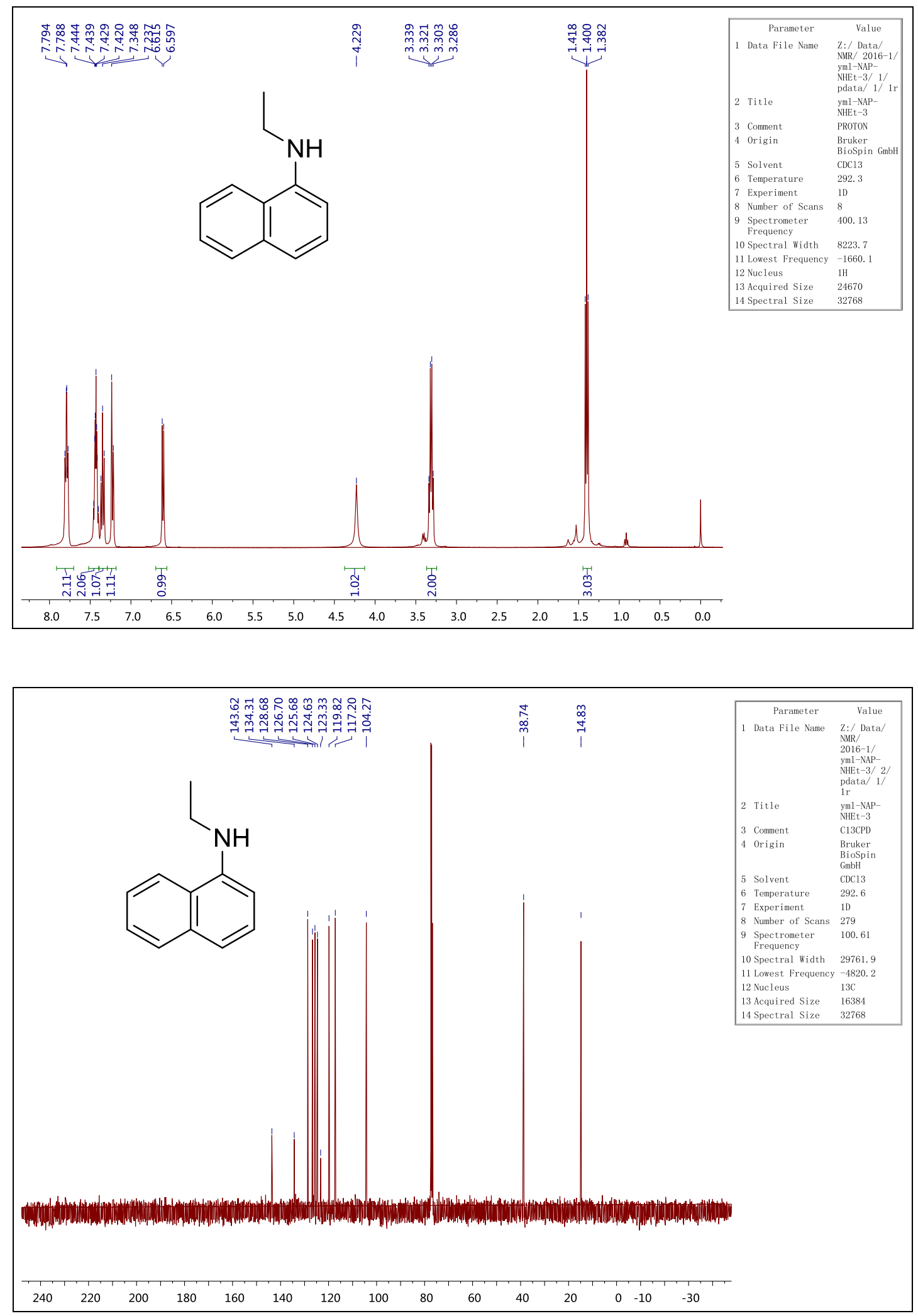
$N$-ethyl-6-methylpyridin-2-amine (2n)
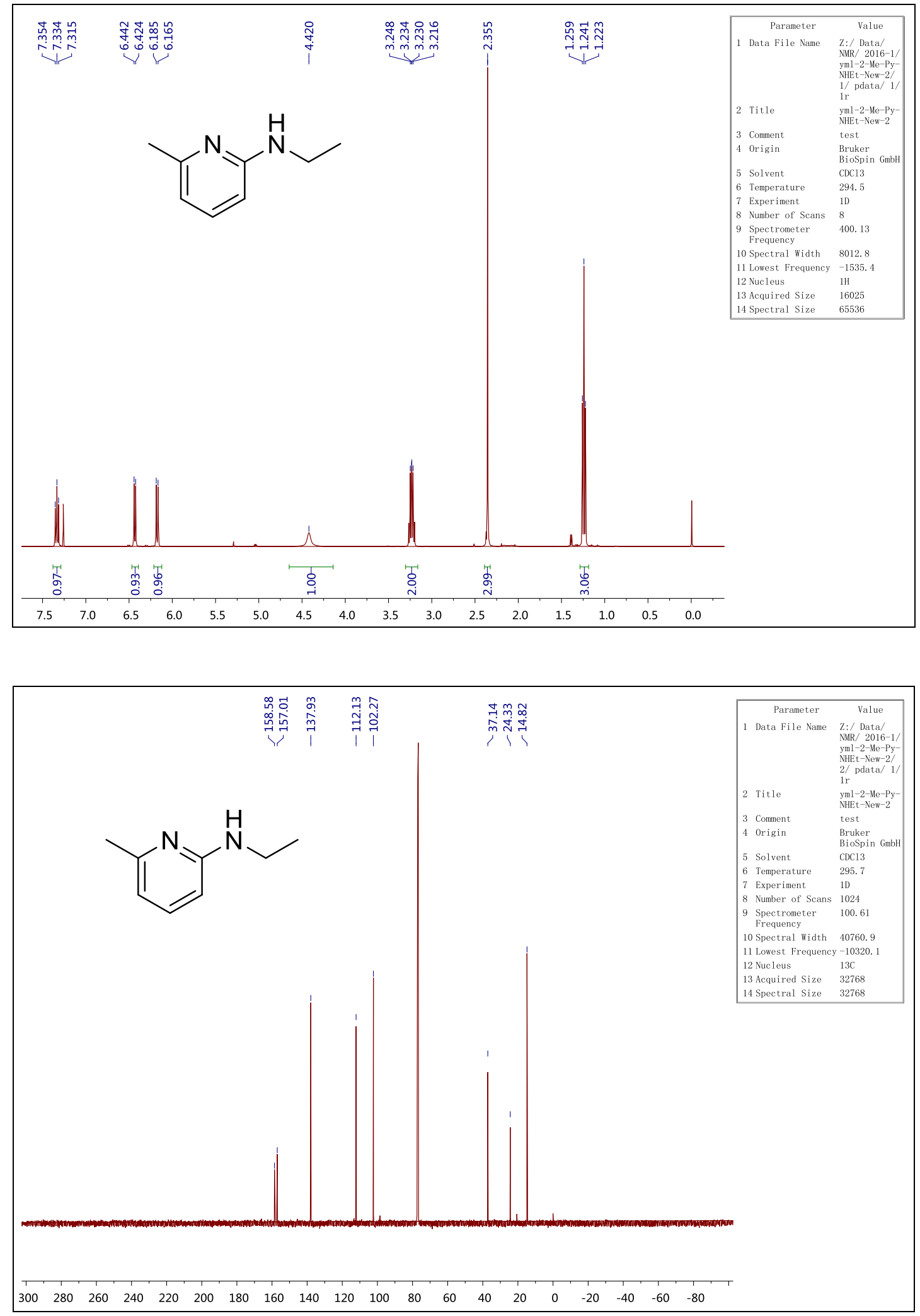
$\mathrm{N}$-methylaniline (2o)
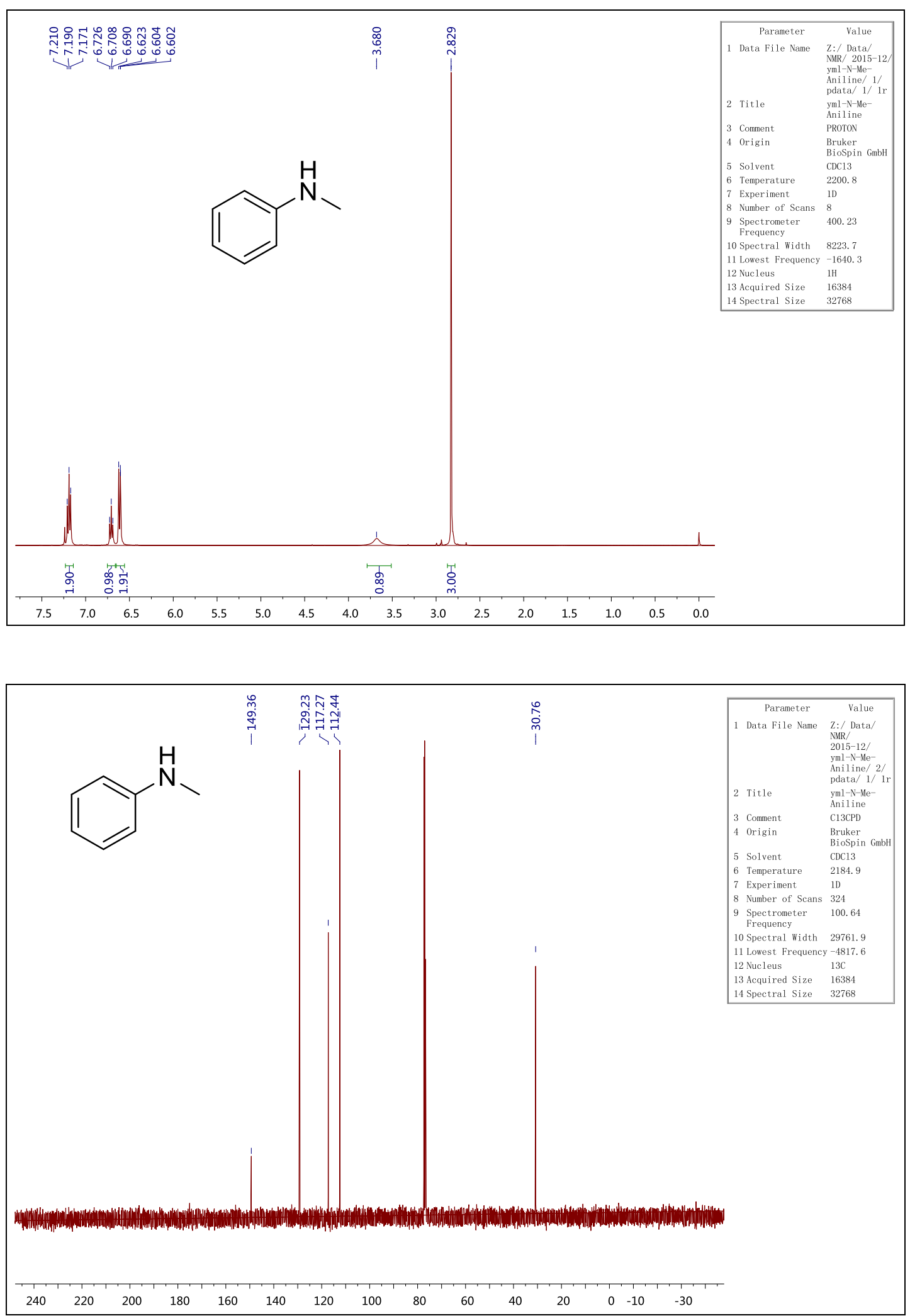
$N$-propylaniline (2p)
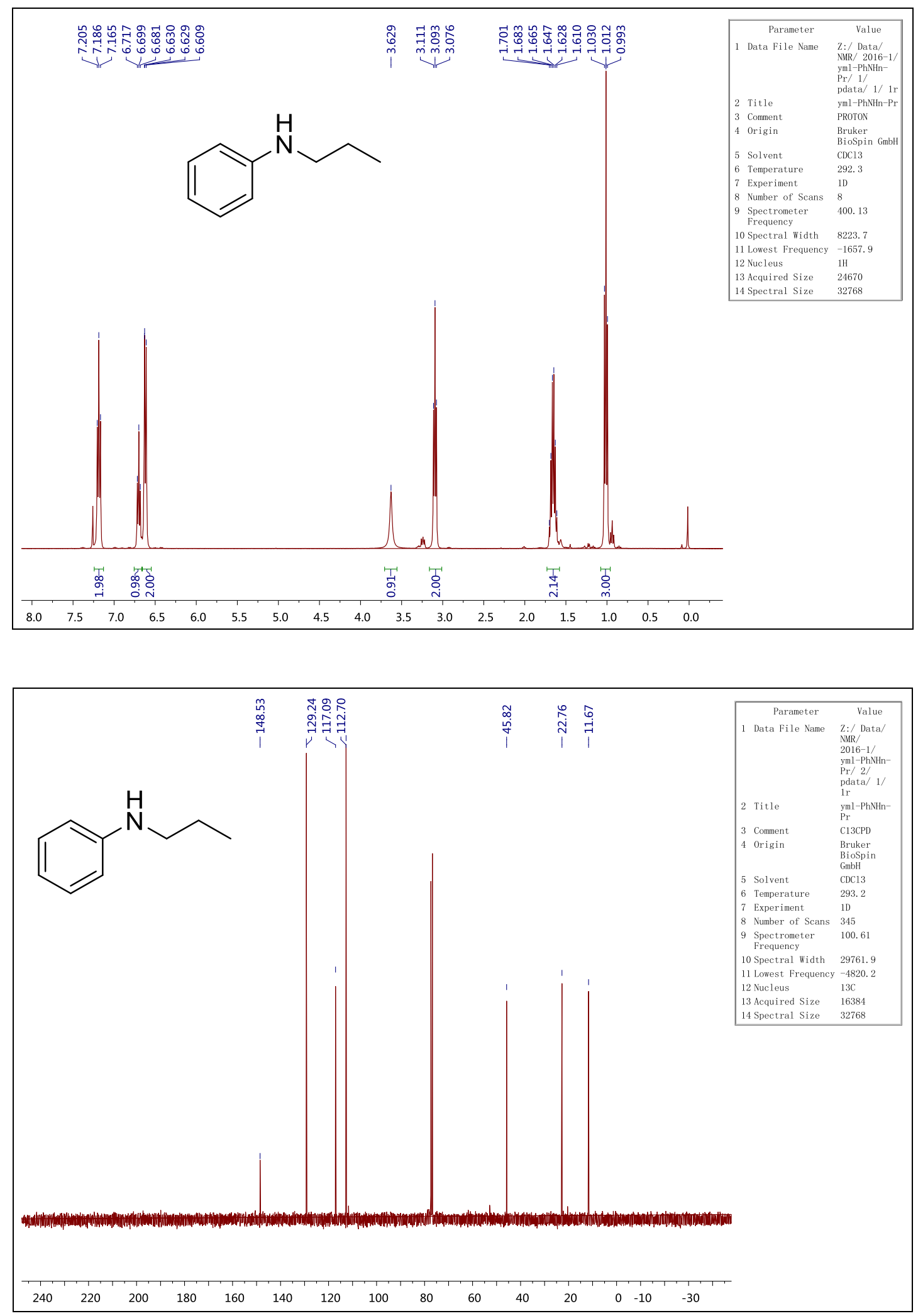
$N$-isobutylaniline (2q)
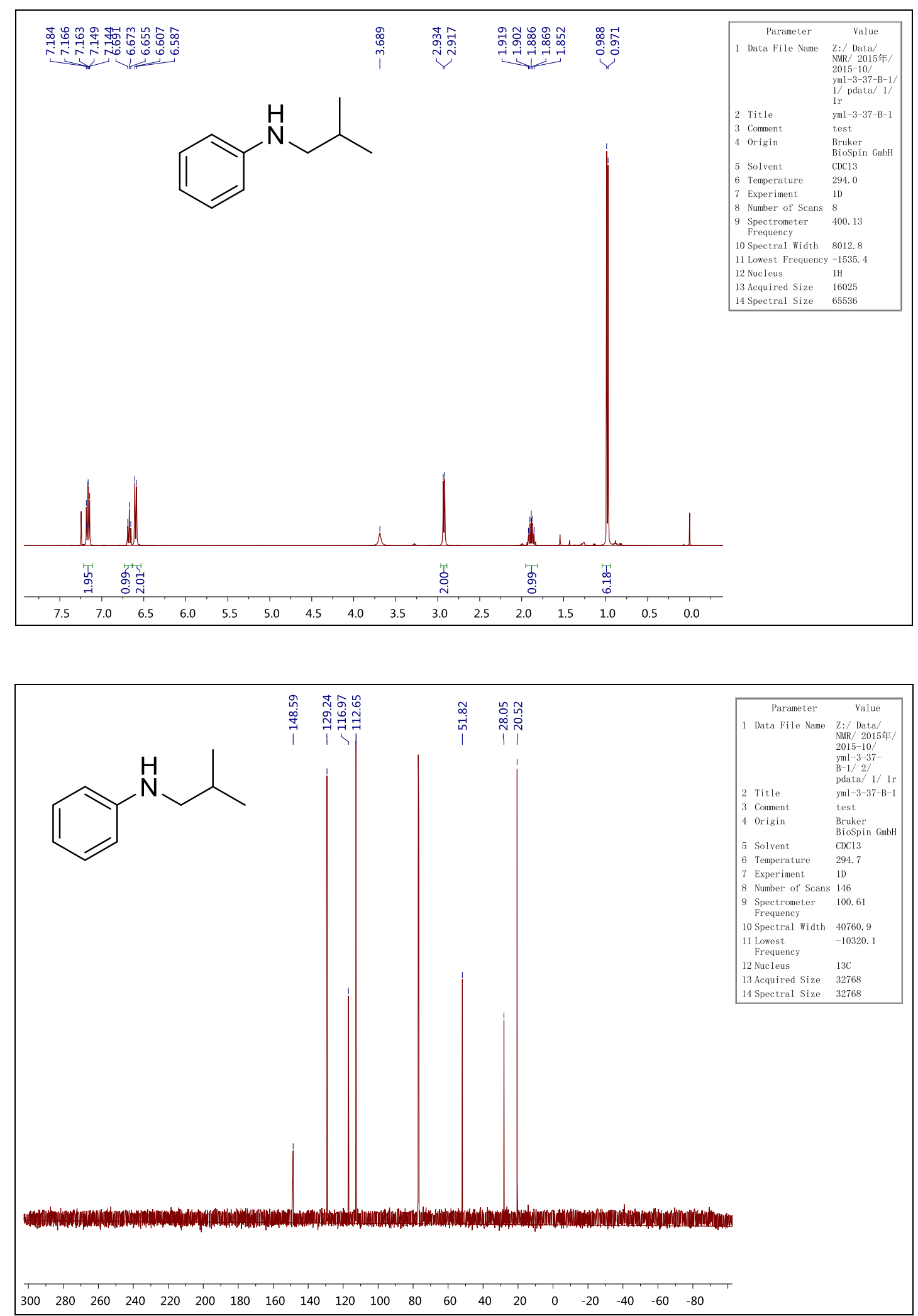
$\mathrm{N}$-dodecylaniline (2r)
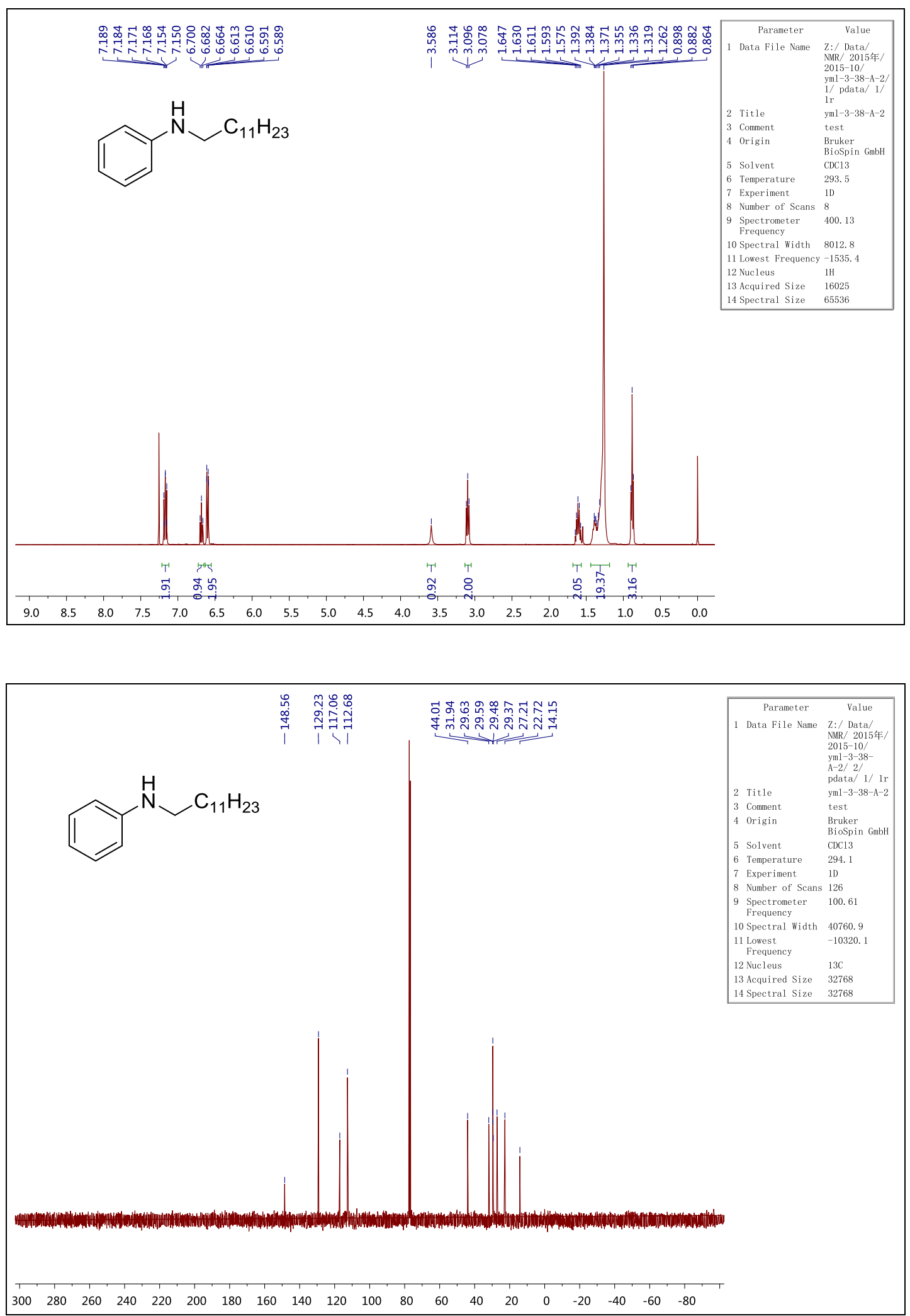
$N$-(2-(benzyloxy)ethyl)aniline (2s)
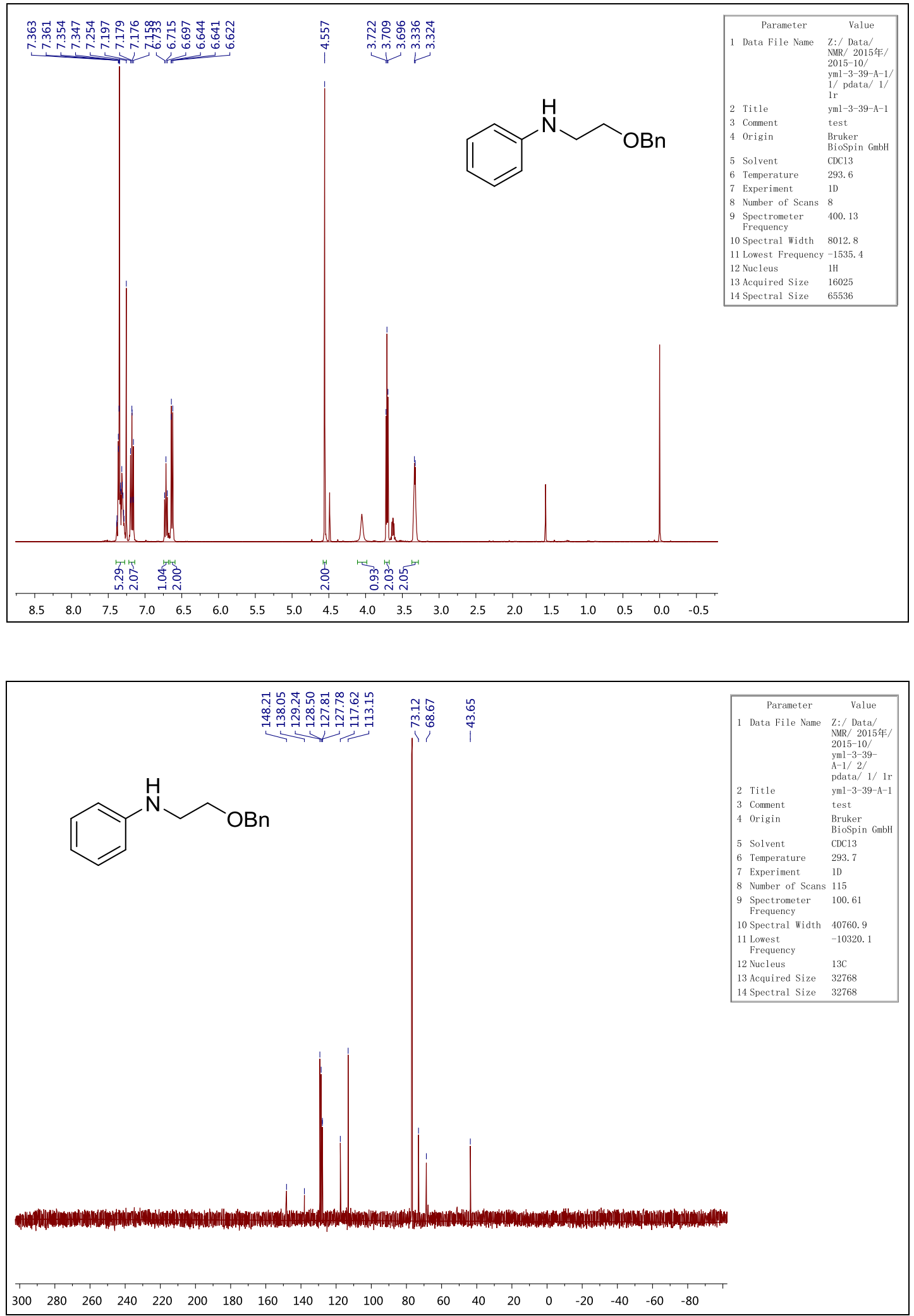
$N$-(2-(thiophen-2-yl)ethyl)aniline (2t)
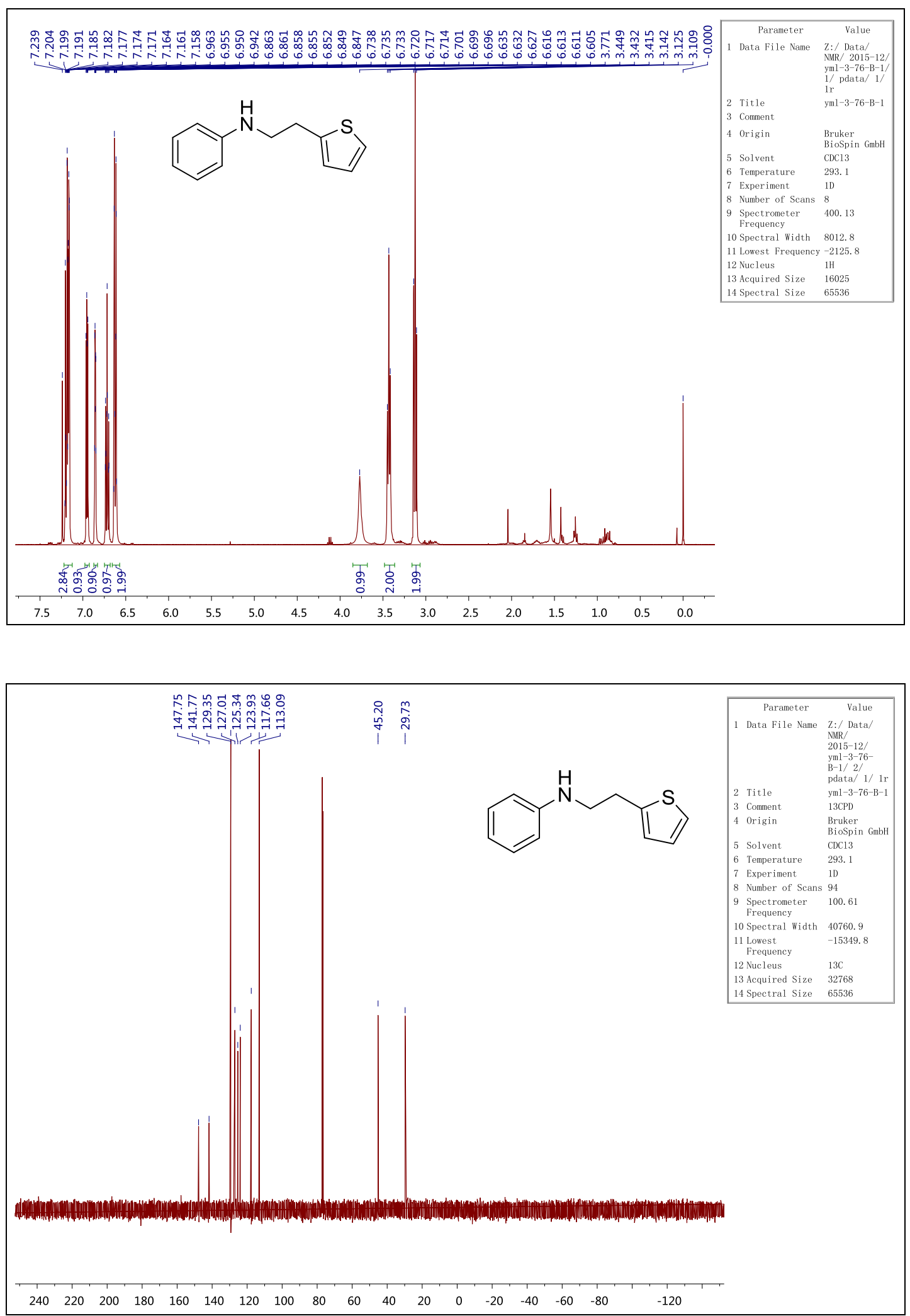


\section{$N$-benzylaniline (2u)}
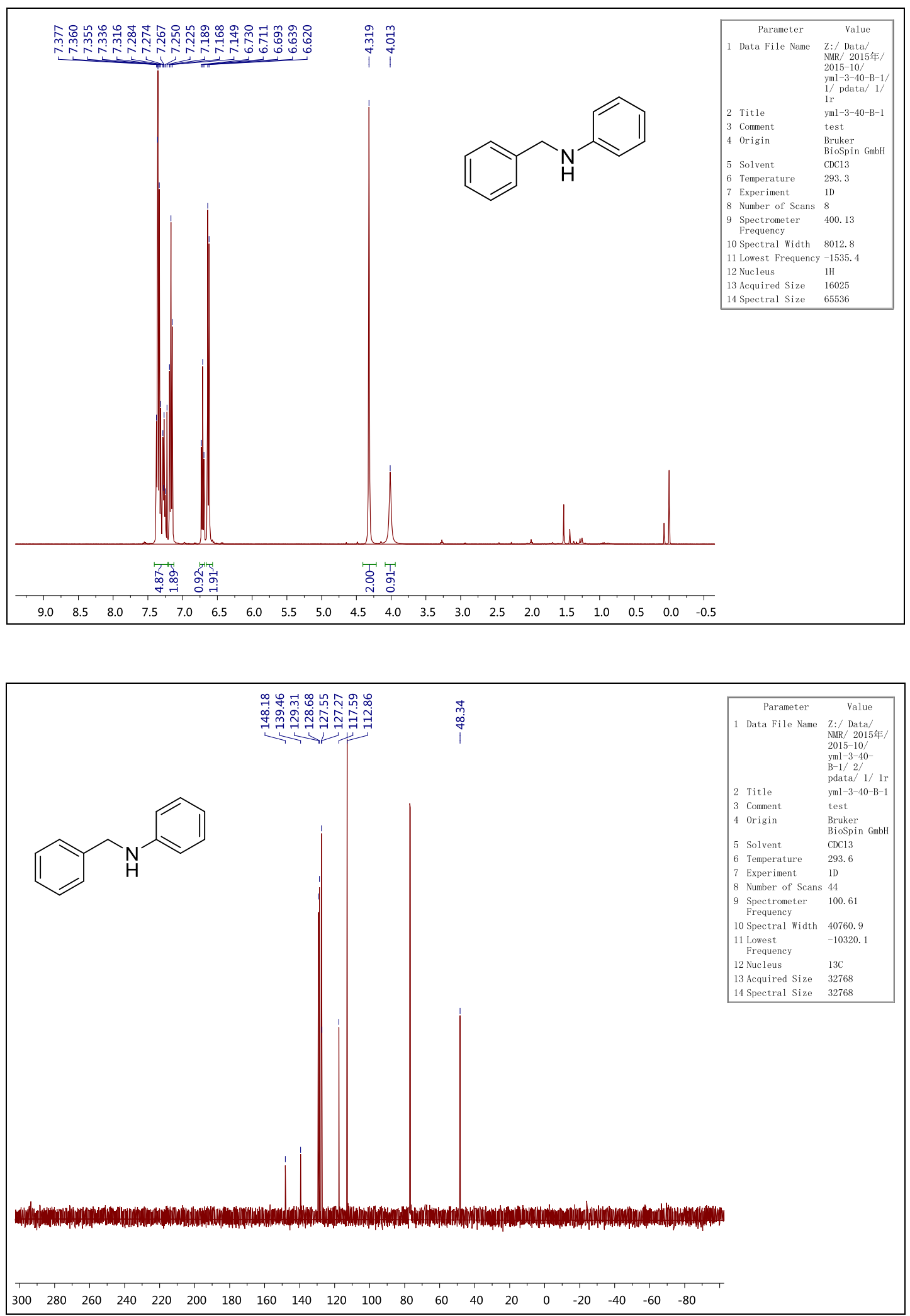
$N$-(furan-2-ylmethyl)aniline (2v)
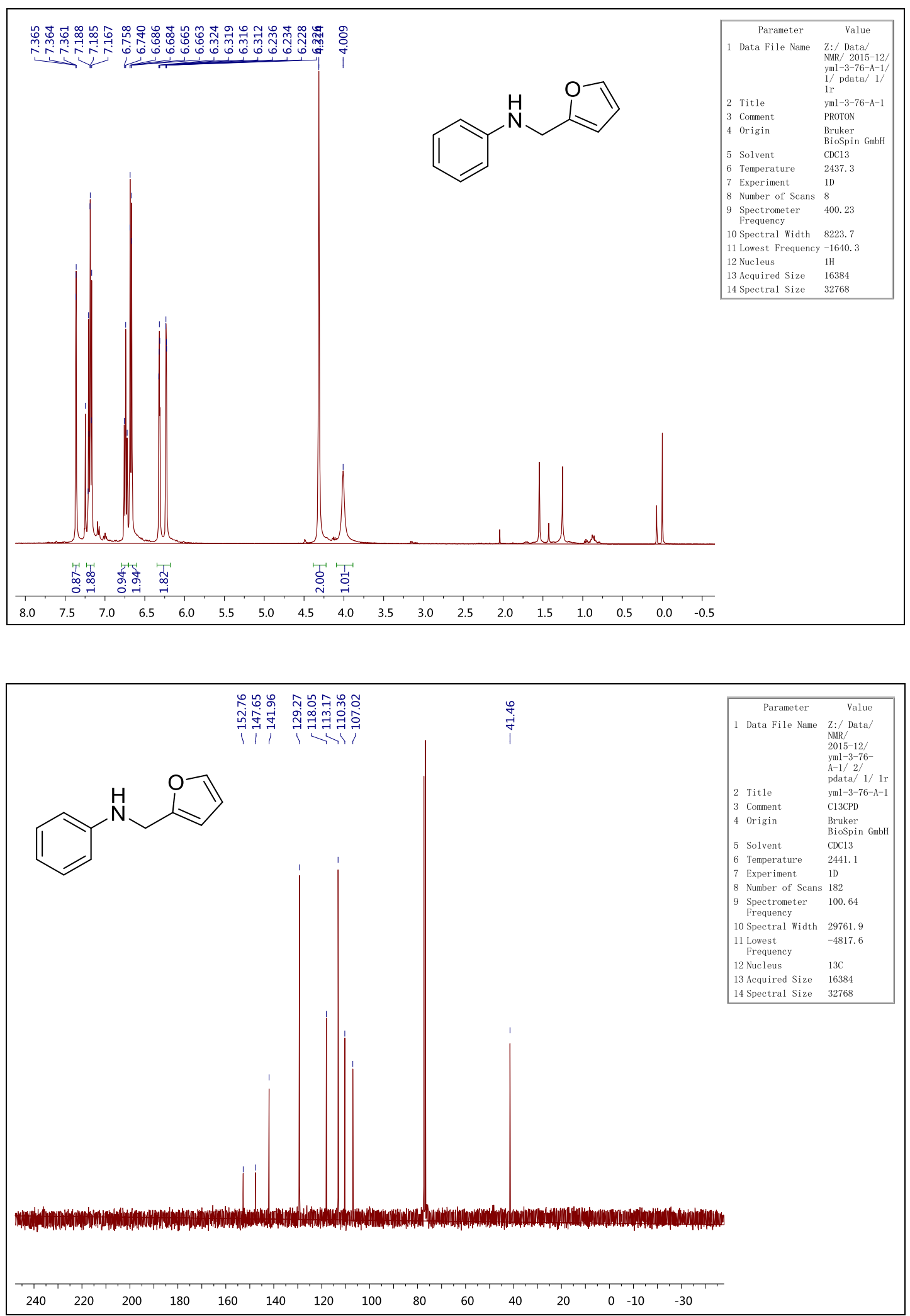